\title{
Preprint
}

\section{Roe-type schemes for shallow water magnetohydrodynamics with hyperbolic divergence cleaning}

\author{
Friedemann Kemm*
}

October 8, 2018

\begin{abstract}
We discuss Roe-type linearizations for non-conservative shallow water magnetohydrodynamics without and with hyperbolic divergence cleaning.
\end{abstract}

\section{Introduction}

Hyperbolic conservation laws are usually equipped with additional conditions. Most important is the existence of a convex entropy-in the case of shallow water the energy-which singles out the physically relevant solution from of the large set of possible weak solutions. Sometimes, especially when there is no convex entropy, or the system degenerates into a weakly or resonant hyperbolic system like shallow water magnetohydrodynamics (SMHD), other laws have to be included to find physical solutions. In the first case (convex entropy), the additional law is introduced to accommodate an additional variable, namely the entropy, which depends on the state variables, but is no state variable itself like the energy in shallow water flows. In the latter case, we have additional partial differential equations for the state variables themselves. In the first case, the additional law is a partial differential equation or inequality of evolution type, usually a conservation law itself, in the latter, it is a first order non-evolutionary constraint. These additional constraints are, as Dafermos points out [7, 8], involutions for the underlying system of conservation laws. So the resulting system, which includes both the evolution system and the condition, has

*Brandenburg University of Technology, Platz der Deutschein Einheit 1, 03046 Cottbus, Germany, kemm@math.tu-cottbus.de 
more equations than unknowns. If the involution is satisfied by the initial state for the evolution equations, it is satisfied by the solution of the evolution system for all times. Thus, in the continuous setting, the constraint is merely a condition for the initial state. The equations of shallow water magnetohydrodynamics (SMHD) are of this latter type, which is a property inherited from full MHD.

To cope with such constraints, there are different approaches in the literature. First, there are approaches, which are designed to model the constraint numerically. Many of them are done on staggered grids [1,2,11]. Some newer approaches also work on collocated grids [12, 13, 26-28, 37, 40, 41, 43] or in the context of Discontinuous-Galerkin schemes [3]. Usually this class of schemes is referred to as constrained transport (CT). Applications to SMHD were provided, e. g., by de Sterck [39] and Rossmanith [36]. The basic strategy for most of these CT-methods is to perform a time step for the conservative evolution equations (standard model). In a second step, the magnetic field is recomputed on the basis of a different equation, quite often on the evolution equation for the vector potential of the magnetic field. As a consequence, the evolution of the magnetic field is, at least formally, non-conservative. An alternative approach is the one provided by Fey and Torrilhon [40,41]. It is based on averaging fluxes of neighbouring Riemann problems, a strategy that is well known to heavily increase numerical viscosity. With the other CTmethods, it shares the drawback that a change in the underlying scheme-e. g. increasing the order of accuracy-also requires a new development of the constraint preserving strategy. Therefore, we concentrate on a different approach.

A second family of schemes is based on a modification of the system of partial differential equations which makes up the constraint part of the evolution system itself. In the context of plasma physics, a popular approach is to transport the involution term, in this case the divergence of the magnetic field, with the flow velocity. This was first suggested for numerical simulations of full MHD by Brackbill and Barnes [4] and put forward by employing Godunovs full symmetrizable form ${ }^{1}$ of the MHD-equations [15] by Powell et al. [32, 33]. In [12, 13, 43], this approach is even combined with constrained transport. Another possibility is to apply a kind of a Generalized Lagrange Multiplier (GLM) approach [31], a method which can appear in several variants: a Hodge-projection scheme, a parabolic treatment of the involution term as was suggested by Marder [25], a hyperbolic system - the involution term is radiated with an artificial speed out of the computational domain [29,30] - or it results in a treatment of the involution in the manner of a telegraph equation [9, 22] (Crockett et al. [6] even combine the Marder approach with a Hodge-projection method.) In the context of electromagnetic models and plasma physics these approaches are usually referred to as divergence cleaning. Although possible in principle, the combination of the transport of the involution with the hyperbolic GLM approach is, to our knowledge, not yet described in the literature 2 .

\footnotetext{
${ }^{1}$ It is interesting to note that this form was first discovered by Godunov [15] as symmetrizable form of MHD and then rediscovered by Powell et al. [32] as Galilean invariant form of MHD. Since it has an entropy [ 8. 15], one would not need any involution for the system.

${ }^{2}$ But it seems to be in practical use, as we can conclude from several oral communications in the last years.
} 
It is worth noting that, as Brackbill and Barnes [4] point out, the standard system of MHD is deduced from physical principles together with the assumption that the magnetic field is solenoidal. Without this a priori assumption, one would simply end up with the Powell system. As we pointed out in our earlier work [22-24], the derivation of the Maxwell equations themselves without the neglect of magnetic charge and magnetic current, just on the basis of physical symmetries, would lead to the standard Maxwell equations augmented by the terms of the hyperbolic GLM correction. Thus, from the physical point of view, it is desirable to employ MHD (and SMHD) equations which are based on the combination of Powell and hyperbolic GLM.

In this paper, we develop (and test) Roe-type schemes for hyperbolic systems of SMHD equations. For the conservative standard equations, as introduced by Gilman [14], the characteristic analysis was performed by de Sterck [38] and a Roe-type scheme developed by Rossmanith [36]. Based on this preparatory work, we investigate the hyperbolic structure of the extended SMHD systems, i. e. with Powell correction and/or hyperbolic GLM correction, in the following section. In Section 3 we discuss possibilities to get Roe matrices for these systems. This again will be based on the work by Rossmanith [36]. In Section 3 the resulting schemes are tested at hand of two prototypical test cases and compared to the scheme for the standard equations. Furthermore, we also cover the choice for the artificial wave speed and the numerical viscosity of the waves which radiate the divergence errors.

\section{The governing equations, combining Powell with GLM-correction}

Here, we discuss the shallow water MHD (SMHD) equations with and without Powell and GLM-correction and their eigensystems. The eigensystems are needed in later sections to set up the Roe-type schemes for the different SMHD models.

\subsection{Conservative equations}

Before we introduce the GLM-correction, we discuss the standard equations and their modification according to Powell. The latter has the desirable property to be fully hyperbolic and not only resonant hyperbolic.

\subsubsection{Without divergence cleaning}

In [14], Gilman argues that the classical "shallow water" equations of geophysical fluid dynamics should be useful for studying the global dynamics of the solar tachocline and demonstrates the existence of an MHD analogon that would allow taking into account the strong toroidal magnetic field likely to be present there. So he presents a derivation 
analogous to that for the classical shallow water equations and comes up with the following system of shallow water magnetohydrodynamics (SMHD) equations

$$
\begin{aligned}
h_{t}+\nabla \cdot[h \boldsymbol{v}] & =0, \\
(h \boldsymbol{v})_{t}+\nabla \cdot\left[h \boldsymbol{v} \circ \boldsymbol{v}-h \boldsymbol{B} \circ \boldsymbol{B}+\frac{g h^{2}}{2} \boldsymbol{I}\right] & =\mathbf{0}, \\
(h \boldsymbol{B})_{t}-\nabla \times[\boldsymbol{v} \times(h \boldsymbol{B})] & =\mathbf{0}, \\
\nabla \cdot(h \boldsymbol{B}) & =0,
\end{aligned}
$$

which inherits most of its behaviour from the original MHD-system. The main difference is that, due to the averaging over the third space dimension, the magnetic field $\boldsymbol{B}$ is now replaced by $h \boldsymbol{B}$, where $h$ denotes the height of the present fluid layer, and $g$ is the gravitational constant. Again, the last equation (4) is a constraint for the evolution system (1) - (3) and does not contribute to the evolution of the solution. For the hyperbolic structure, we only consider the evolution system (1) - (3).

Because the system is rotationally invariant, we therefore consider in the following the flux Jacobian only for the first space-direction, which reads as

$$
\boldsymbol{A}_{\text {standard }}=\left(\begin{array}{ccccc}
0 & 1 & 0 & 0 & 0 \\
-u^{2}+B_{1}^{2}+g h & 2 u & 0 & -2 B_{1} & 0 \\
-u v+B_{1} B_{2} & v & u & -B_{2} & -B_{1} \\
0 & 0 & 0 & 0 & 0 \\
v B_{1}-u B_{2} & B_{2} & -B_{1} & -v & u
\end{array}\right) .
$$

The wave speeds, i. e. the eigenvalues of the flux Jacobian in the first space direction, are

$$
\lambda_{1}=u-c_{g}, \quad \lambda_{2}=u-\left|B_{1}\right|, \quad \lambda_{3}=0, \quad \lambda_{4}=u+\left|B_{1}\right|, \quad \lambda_{5}=u+c_{g}
$$

with the magneto-gravitational speed

$$
c_{g}=\sqrt{B_{1}^{2}+g h},
$$

which, for a vanishing magnetic field, would coincide with the celerity $c_{g}$ of the shallow water equations. The third eigenvalue $\lambda_{3}=0$ indicates that the system is not Galilean invariant. For the other wave speeds,

$$
\lambda_{1} \leq \lambda_{2} \leq \lambda_{4} \leq \lambda_{5}
$$

is satisfied for any state $\boldsymbol{q}$. If the first component of the magnetic field vanishes, $\lambda_{2}$ and $\lambda_{4}$ coincide. Thus, the system is not strictly hyperbolic. To decide if the system is hyperbolic or weakly/resonant hyperbolic, one has to consider the eigenvectors. The right eigenvectors 
$\operatorname{are} \mathrm{S}^{3}$

$$
\boldsymbol{r}_{1}=\left(\begin{array}{c}
1 \\
u-c_{g} \\
v \\
0 \\
B_{2}
\end{array}\right), \quad \boldsymbol{r}_{5}=\left(\begin{array}{c}
1 \\
u+c_{g} \\
v \\
0 \\
B_{2}
\end{array}\right)
$$

for the magneto-gravitational waves,

$$
\boldsymbol{r}_{2}=\left(\begin{array}{c}
0 \\
0 \\
1 \\
0 \\
\operatorname{sgn}\left(B_{1}\right)
\end{array}\right), \quad \boldsymbol{r}_{4}=\left(\begin{array}{c}
0 \\
0 \\
1 \\
0 \\
-\operatorname{sgn}\left(B_{1}\right)
\end{array}\right)
$$

for the Alfven waves, and

$$
\boldsymbol{r}_{3}=\left(\begin{array}{c}
2 B_{1}\left(u-B_{1}\right)\left(u+B_{1}\right) \\
\left(v B_{1}-u B_{2}\right)\left(u-B_{1}\right)\left(u+B_{1}\right)+g h\left(v B_{1}+u B_{2}\right) \\
-\left(u-c_{g}\right)\left(u+c_{g}\right)\left(u-B_{1}\right)\left(u+B_{1}\right) \\
\left(B_{1} B_{2}-u v\right)\left(u-B_{1}\right)\left(u+B_{1}\right)+g h\left(B_{1} B_{2}+u v\right)
\end{array}\right) .
$$

for the remaining wave. If for any $k \neq 3$ the eigenvalue $\lambda_{k}$ vanishes, the system is only resonant hyperbolic.

The according left eigenvalues are

$$
\begin{aligned}
& l_{1}=\frac{1}{2 c_{g}}\left(u+c_{g},-1,0, \frac{2 B_{1}}{u-c_{g}}, 0\right) \\
& l_{2}=\frac{1}{2}\left(-\operatorname{sgn}\left(B_{1}\right) B_{2}-v, 0,1, \frac{-\operatorname{sgn}\left(B_{1}\right) v-B_{2}}{u-\left|B_{1}\right|}, \operatorname{sgn}\left(B_{1}\right)\right) \\
& l_{3}=\left(0,0,0,-\frac{1}{\left(u-c_{g}\right)\left(u+c_{g}\right)\left(u-B_{1}\right)\left(u+B_{1}\right)}, 0\right) \\
& l_{4}=\frac{1}{2}\left(\operatorname{sgn}\left(B_{1}\right) B_{2}-v, 0,1, \frac{\operatorname{sgn}\left(B_{1}\right) v-B_{2}}{u-\left|B_{1}\right|},-\operatorname{sgn}\left(B_{1}\right)\right) \\
& l_{5}=-\frac{1}{2 c_{g}}\left(u-c_{g},-1,0, \frac{2 B_{1}}{u+c_{g}}, 0\right)
\end{aligned}
$$

These are used in the implementation of the numerical schemes in clawpack [5] to perform the numerical experiments for shallow water MHD in Section 4. There, we also employ

\footnotetext{
${ }^{3}$ Note that the eigensystem given in this section deviates from the system given by de Sterck [38, 39]. The reason is that de Sterck investigates the system with the so called Powell correction which will be discussed in Section 2.2.1.
} 
solvers which are based on the assumption of one-dimensional physics for the flux normal to a cell face. In $1 \mathrm{~d}$, equation (4) would require $h B_{1}$ to be constant. Together with the 1d-evolution equation, this would mean that $h B_{1}$ is just a constant parameter. Thus, one equation can be dropped from the system (1) - (3). As a consequence, the third characteristic field, $\lambda_{3}$ together with its eigenvectors, no longer exists. For the other eigenvectors, the third component should be omitted. As we demonstrated in [20], the application of solvers based on $1 \mathrm{~d}$ physics in a $2 \mathrm{~d}$ situation leads to the failure of the scheme due to excessive divergence errors.

\subsubsection{With GLM-correction}

If we introduce the GLM-correction as it is described in [9] for MHD and adopted to SMHD in [23,24], we have to add the gradient of $h \psi$ to the evolution of the magnetic field and convert the divergence constraint (cf. equation (4)) into the time evolution for $h \psi$ (cf. equation (17), where $\psi$ is an artificial quantity. Formally, it is the potential of the magnetic current. In physics, the magnetic field is solenoidal. Thus, there is no magnetic current, and $\psi$ is a mere constant. The equations then read as

$$
\begin{aligned}
h_{t}+\nabla \cdot[h \boldsymbol{v}] & =0, \\
(h \boldsymbol{v})_{t}+\nabla \cdot\left[h \boldsymbol{v} \circ \boldsymbol{v}-h \boldsymbol{B} \circ \boldsymbol{B}+\frac{g h^{2}}{2} \boldsymbol{I}\right] & =\mathbf{0}, \\
(h \boldsymbol{B})_{t}-\nabla \times[\boldsymbol{v} \times(h \boldsymbol{B})]+\nabla(h \psi) & =\mathbf{0}, \\
(h \psi)_{t}+c_{\psi}^{2} \nabla \cdot(h \boldsymbol{B}) & =0,
\end{aligned}
$$

where $c_{\psi}$ is a constant artificial wave speed. The goal is to transport the (nonphysical) magnetic monopoles with that speed out of the computational domain. Again, we consider the flux Jacobian for the first space direction:

$$
\boldsymbol{A}_{\mathrm{GLM}}=\left(\begin{array}{cccccc}
0 & 1 & 0 & 0 & 0 & 0 \\
-u^{2}+B_{1}^{2}+g h & 2 u & 0 & -2 B_{1} & 0 & 0 \\
-u v+B_{1} B_{2} & v & u & -B_{2} & -B_{1} & 0 \\
0 & 0 & 0 & 0 & 0 & 1 \\
v B_{1}-u B_{2} & B_{2} & -B_{1} & -v & u & 0 \\
0 & 0 & 0 & c^{2} & 0 & 0
\end{array}\right) .
$$

Compared to $\boldsymbol{A}_{\text {standard }}$ (cf. equation (5)), a sixth row and column was added. However, the eigensystem is changed considerably. The eigenvectors are eigenvalues to

$$
\lambda_{1}=-c, \quad \lambda_{2},=u-c_{g}, \quad \lambda_{3}=u-\left|B_{1}\right|, \quad \lambda_{4}=u+\left|B_{1}\right|, \quad \lambda_{5},=u+c_{g}, \quad \lambda_{6}=c,
$$

just as expected. But the eigenvectors are more complicated. The right eigenvectors for the Alfven waves are only augmented by a vanishing sixth component and renumbered according to (19). The same is true for the magnetogravitational waves. The right 
eigenvectors for the waves transporting the divergence errors outside of the computational domain are

$$
\boldsymbol{r}_{1}=\left(\begin{array}{c}
-\frac{2 B_{1}}{c_{\psi}\left(c_{g}^{2}-\left(u+c_{\psi}\right)^{2}\right)} \\
\frac{2 B_{1}}{c_{g}^{2}-\left(u+c_{\psi}\right)^{2}} \\
-\frac{\left(u+c_{\psi}\right)\left[B_{2}\left(\left(u+c_{\psi}\right)^{2}+c_{g}^{2}\right)-B_{1} v\left(u+c_{\psi}\right)\right]+v B_{1}\left(2 B_{1}^{2}-c_{g}^{2}\right)}{c_{\psi}\left(B_{1}^{2}-\left(u+c_{\psi}\right)^{2}\right)\left(c_{g}^{2}-\left(u+c_{\psi}\right)^{2}\right)} \\
-\frac{1}{c} \\
-\frac{\left(u+c_{\psi}\right)\left[v\left(\left(u+c_{\psi}\right)^{2}+c_{g}^{2}\right)-B_{1} B_{2}\left(u+c_{\psi}\right)\right]+B_{1} B_{2}\left(2 B_{1}^{2}-c_{g}^{2}\right)}{c_{\psi}\left(B_{1}^{2}-\left(u+c_{\psi}\right)^{2}\right)\left(c_{g}^{2}-\left(u+c_{\psi}\right)^{2}\right)} \\
1
\end{array}\right)
$$

and

$$
\boldsymbol{r}_{6}=\left(\begin{array}{c}
\frac{2 B_{1}}{c_{\psi}\left(c_{g}^{2}-\left(u-c_{\psi}\right)^{2}\right)} \\
\frac{2 B_{1}}{c_{g}^{2}-\left(u-c_{\psi}\right)^{2}} \\
\frac{\left(u-c_{\psi}\right)\left[B_{2}\left(\left(u-c_{\psi}\right)^{2}+c_{g}^{2}\right)-B_{1} v\left(u-c_{\psi}\right)\right]+v B_{1}\left(2 B_{1}^{2}-c_{g}^{2}\right)}{c_{\psi}\left(B_{1}^{2}-\left(u-c_{\psi}\right)^{2}\right)\left(c_{g}^{2}-\left(u-c_{\psi}\right)^{2}\right)} \\
\frac{1}{c} \\
\frac{\left(u-c_{\psi}\right)\left[v\left(\left(u-c_{\psi}\right)^{2}+c_{g}^{2}\right)-B_{1} B_{2}\left(u-c_{\psi}\right)\right]+B_{1} B_{2}\left(2 B_{1}^{2}-c_{g}^{2}\right)}{c_{\psi}\left(B_{1}^{2}-\left(u-c_{\psi}\right)^{2}\right)\left(c_{g}^{2}-\left(u-c_{\psi}\right)^{2}\right)}
\end{array}\right)
$$

On the other hand, the corresponding left eigenvectors for the first and sixth wave are rather simple:

$$
\begin{aligned}
\boldsymbol{l}_{1} & =\frac{1}{2}\left(0,0,0,-c_{\psi}, o, 1\right)^{T} \\
\boldsymbol{l}_{6} & =\frac{1}{2}\left(0,0,0, c_{\psi}, o, 1\right)^{T}
\end{aligned}
$$


The left eigenvectors for the Alfven waves read as

$$
\begin{aligned}
& \boldsymbol{l}_{3}=\frac{\operatorname{sgn}\left(B_{1}\right)}{2}\left(-\operatorname{sgn}\left(B_{1}\right) v-B_{2}, 0, \operatorname{sgn}\left(B_{1}\right),-\frac{\left(B_{1}-\operatorname{sgn}\left(B_{1}\right) u\right)\left(B_{2}+\operatorname{sgn}\left(B_{1}\right) v\right)}{\left(u-\operatorname{sgn}\left(B_{1}\right) B_{1}\right)^{2}-c_{\psi}^{2}}, 1,-\frac{v+\operatorname{sgn}\left(B_{1}\right) B_{2}}{\left(u-\operatorname{sgn}\left(B_{1}\right) B_{1}\right)^{2}-c_{\psi}^{2}}\right)^{T}, \\
& \boldsymbol{l}_{4}=\frac{-\operatorname{sgn}\left(B_{1}\right)}{2}\left(\operatorname{sgn}\left(B_{1}\right) v-B_{2}, 0,-\operatorname{sgn}\left(B_{1}\right),-\frac{\left(B_{1}+\operatorname{sgn}\left(B_{1}\right) u\right)\left(B_{2}-\operatorname{sgn}\left(B_{1}\right) v\right)}{\left(u+\operatorname{sgn}\left(B_{1}\right) B_{1}\right)^{2}-c_{\psi}^{2}}, 1,-\frac{v-\operatorname{sgn}\left(B_{1}\right) B_{2}}{\left(u+\operatorname{sgn}\left(B_{1}\right) B_{1}\right)^{2}-c_{\psi}^{2}}\right)^{T} .
\end{aligned}
$$

The left eigenvectors for the magneto-gravitational waves are

$$
\begin{aligned}
& \boldsymbol{l}_{2}=\frac{1}{2 c_{g}}\left(u+c_{g},-1,0,-\frac{2 B_{1}\left(u-c_{g}\right)}{c_{\psi}^{2}-\left(u-c_{g}\right)^{2}}, 0,-\frac{2 B_{1}}{c_{\psi}^{2}-\left(u-c_{g}\right)^{2}}\right), \\
& \boldsymbol{l}_{5}=\frac{1}{2 c_{g}}\left(-\left(u-c_{g}\right), 1,0, \frac{2 B_{1}\left(u+c_{g}\right)}{c_{\psi}^{2}-\left(u+c_{g}\right)^{2}}, 0, \frac{2 B_{1}}{c_{\psi}^{2}-\left(u+c_{g}\right)^{2}}\right) .
\end{aligned}
$$

The main differences to the corresponding eigenvectors for the standard system (cf. equation (13)) are the changes in the fourth component and the new sixth component.

\subsection{Non-conservative equations}

\subsubsection{The Powell correction}

The above systems lack Galilean invariance. This can be restored by giving up conservation, e. $g$. for the standard system (1) - (3) by replacing the right hand side of $(1)-(3)$ with

$$
-(0,(\nabla \cdot(h \boldsymbol{B})) \boldsymbol{B},(\nabla \cdot(h \boldsymbol{B})) \boldsymbol{v})^{T}
$$

which results in the so called Powell-correction.

Thus, the system matrix in the first space direction reads as

$$
A_{\text {Powell }}=\left(\begin{array}{ccccc}
0 & 1 & 0 & 0 & 0 \\
-u^{2}+B_{1}^{2}+g h & 2 u & 0 & -B_{1} & 0 \\
-u v+B_{1} B_{2} & v & u & 0 & -B_{1} \\
0 & 0 & 0 & u & 0 \\
v B_{1}-u B_{2} & B_{2} & -B_{1} & 0 & u
\end{array}\right) .
$$

Now the third Eigenvalue is no longer zero but

$$
\lambda_{3}=u
$$


The right eigenvectors $\boldsymbol{r}_{1}, \boldsymbol{r}_{2}, \boldsymbol{r}_{4}$, and $\boldsymbol{r}_{5}$ remain unchanged, whereas the right eigenvector of the modified eigenvalue reads as

$$
\boldsymbol{r}_{3}=\left(\begin{array}{c}
B_{1} \\
u B_{1} \\
v B_{1} \\
c_{g}^{2} \\
B_{2} B_{1}
\end{array}\right)
$$

which is simpler than for the conservative system. Furthermore it guarantees hyperbolicity of the system. For the left eigenvectors, the only change is in the fourth component, the component connected to $B_{1}$ :

$$
\begin{aligned}
& l_{1}=\frac{1}{2 c_{g}}\left(u+c_{g},-1,0,-\frac{B_{1}}{c_{g}}, 0\right) \\
& l_{2}=\frac{1}{2}\left(-\operatorname{sgn}\left(B_{1}\right) B_{2}-v, 0,1,0, \operatorname{sgn}\left(B_{1}\right)\right) \\
& l_{3}=\left(0,0,0, \frac{1}{c_{g}^{2}}, 0\right) \\
& l_{4}=-\frac{1}{2}\left(\operatorname{sgn}\left(B_{1}\right) B_{2}-v, 0,1,0,-\operatorname{sgn}\left(B_{1}\right)\right) \\
& l_{5}=-\frac{1}{2 c_{g}}\left(u-c_{g},-1,0, \frac{B_{1}}{c_{g}}, 0\right)
\end{aligned}
$$

\subsubsection{Combining Powell- and GLM-correction}

In order to apply the GLM divergence correction to the Powell system (1) - (3), we have to add, in the momentum equation (2), the gradient of $h \psi$ to the flux, and the the conservation equation for $h \psi$

$$
(h \psi)_{t}+\nabla \cdot\left(h \psi v+c^{2} h \boldsymbol{B}\right)=0,
$$

where $c$ is the artificial (constant) wave speed at which the magnetic charges are radiated. Equation (30) means that $\psi$ is transported with the flow, and that it is conserved. The flux 
Jacobian for the first space direction reads as

$$
\boldsymbol{A}_{\text {PGLM-naive }}=\left(\begin{array}{cccccc}
0 & 1 & 0 & 0 & 0 & 0 \\
-u^{2}+B_{1}^{2}+g h & 2 u & 0 & -2 B_{1} & 0 & 0 \\
-u v+B_{1} B_{2} & v & u & -B_{2} & -B_{1} & 0 \\
0 & 0 & 0 & 0 & 0 & 1 \\
v B_{1}-u B_{2} & B_{2} & -B_{1} & -v & u & 0 \\
u \psi & \psi & 0 & c^{2} & 0 & u
\end{array}\right) .
$$

The eigensystem of this matrix is rather awkward. This is mainly due to the transport of the magnetic potential with the flow in equation (30). If we would omit the transport term $h \psi v$ in the flux term in equation (30), at least the eigenvalues would be as expected, and the eigenvectors less complicated, but this is not discussed further here.

If we make the system Galilean invariant, that means, if we subtract

$$
(0,(\nabla \cdot(h \boldsymbol{B})) \boldsymbol{B},(\nabla \cdot(h \boldsymbol{B})) \boldsymbol{v},-h \psi \nabla \cdot \boldsymbol{v})^{T}
$$

from the right hand side of the conservation system, the system matrix for the first space direction becomes

$$
\boldsymbol{A}_{\mathrm{PGLM}}=\left(\begin{array}{cccccc}
0 & 1 & 0 & 0 & 0 & 0 \\
-u^{2}+B_{1}^{2}+g h & 2 u & 0 & -B_{1} & 0 & 0 \\
-u v+B_{1} B_{2} & v & u & 0 & -B_{1} & 0 \\
0 & 0 & 0 & u & 0 & 1 \\
v B_{1}-u B_{2} & B_{2} & -B_{1} & 0 & u & 0 \\
0 & 0 & 0 & c^{2} & 0 & u
\end{array}\right) .
$$

The eigenvalues now are

$\lambda_{1}=u-c, \quad \lambda_{2},=u-c_{g}, \quad \lambda_{3}=u-\left|B_{1}\right|, \quad \lambda_{4}=u+\left|B_{1}\right|, \quad \lambda_{5},=u+c_{g}, \quad \lambda_{6}=u+c$. 
The right eigenvectors are much simpler than for GLM without Powell:

$$
\boldsymbol{r}_{2}=\left(\begin{array}{c}
1 \\
u-c_{g} \\
v \\
0 \\
B_{2} \\
0
\end{array}\right), \quad \boldsymbol{r}_{5}=\left(\begin{array}{c}
1 \\
u+c_{g} \\
v \\
0 \\
B_{2} \\
0
\end{array}\right)
$$

for the magneto-gravitational waves, and

$$
\boldsymbol{r}_{3}=\left(\begin{array}{c}
0 \\
0 \\
1 \\
0 \\
\operatorname{sgn}\left(B_{1}\right) \\
0
\end{array}\right), \quad \boldsymbol{r}_{4}=\left(\begin{array}{c}
0 \\
0 \\
1 \\
0 \\
-\operatorname{sgn}\left(B_{1}\right) \\
0,
\end{array}\right)
$$

for the Alfven waves,which means that compared to the conservative system without GLM-correction only a sixth component with value 0 is appended. For the waves advecting and radiating away the divergence errors, the right eigenvectors are

$$
\boldsymbol{r}_{1}=\left(\begin{array}{c}
-B_{1} \\
-B_{1}(u-c) \\
-B_{1} v \\
c^{2}-c_{g}^{2} \\
-B_{1} B_{2} \\
-c\left(c^{2}-c_{g}^{2}\right)
\end{array}\right), \quad \boldsymbol{r}_{6}=\left(\begin{array}{c}
B_{1} \\
B_{1}(u+c) \\
B_{1} v \\
-\left(c^{2}-c_{g}^{2}\right) \\
B_{1} B_{2} \\
-c\left(c^{2}-c_{g}^{2}\right)
\end{array}\right) .
$$

The first, third and fifth component coincide with the according eigenvector for the Powell 
system without GLM. The left eigenvalues are

$$
\begin{aligned}
& l_{1}=\frac{1}{2 c\left(c^{2}-c_{g}^{2}\right)}(0,0,0, c, 0,-1) \\
& l_{2}=\frac{1}{2 c_{g}}\left(u+c_{g},-1,0, \frac{c_{g} B_{1}}{c^{2}-c_{g}^{2}}, 0,-\frac{B_{1}}{c^{2}-c_{g}^{2}}\right) \\
& l_{3}=\frac{1}{2}\left(-\operatorname{sgn}\left(B_{1}\right) B_{2}-v, 0,1,0, \operatorname{sgn}\left(B_{1}\right), 0\right) \\
& l_{4}=-\frac{1}{2}\left(\operatorname{sgn}\left(B_{1}\right) B_{2}-v, 0,1,0,-\operatorname{sgn}\left(B_{1}\right), 0\right) \\
& l_{5}=-\frac{1}{2 c_{g}}\left(u-c_{g},-1,0,-\frac{c_{g} B_{1}}{c^{2}-c_{g}^{2}}, 0,-\frac{B_{1}}{c^{2}-c_{g}^{2}}\right) \\
& l_{1}=\frac{1}{2 c\left(c^{2}-c_{g}^{2}\right)}(0,0,0, c, 0,-1) .
\end{aligned}
$$

In order to avoid resonance, we have to make sure that $c \neq c_{g}$. Otherwise, the first and second as well as the fifth and sixth eigenvectors would coincide. For practical use, it is desirable to have $c>c_{g}$ so that $\lambda_{1}<\lambda_{2}$ and $\lambda_{6}>\lambda_{5}$, which ensures that the waves transporting the unphysical magnetic charge interfere with the other waves as little as possible.

Since the first and the sixth wave do not transport any physical feature, but are intended to transport divergence errors out of the computational domain, there is no real need to make them Galilean invariant. In fact, we could keep the eigenvectors and change the eigenvalues to

$$
\lambda_{1}=-c, \quad \lambda_{2},=u-c_{g}, \quad \lambda_{3}=u-\left|B_{1}\right|, \quad \lambda_{4}=u+\left|B_{1}\right|, \quad \lambda_{5},=u+c_{g}, \quad \lambda_{6}=c .
$$

If the artificial wave speed $c$ is larger than any physical wave speed, we not only avoid resonance, but also make sure that the errors are quickly transported out of the computational domain. The corresponding system matrix for the first space direction then reads as

$$
\boldsymbol{A}_{\mathrm{PGLM}-\mathrm{nG}}=\left(\begin{array}{cccccc}
0 & 1 & 0 & \frac{u B_{1}}{c^{2}-c_{g}^{2}} & 0 & 0 \\
-u^{2}+B_{1}^{2}+g h & 2 u & 0 & -B_{1}+\frac{u^{2} B_{1}}{c^{2}-c_{g}^{2}} & 0 & \frac{u B_{1}}{c^{2}-c_{g}^{2}} \\
-u v+B_{1} B_{2} & v & u & \frac{u v B_{1}}{c^{2}-c_{g}^{2}} & -B_{1} & 0 \\
0 & 0 & 0 & 0 & 0 & 1 \\
v B_{1}-u B_{2} & B_{2} & -B_{1} & \frac{u B_{1} B_{2}}{c^{2}-c_{g}^{2}} & u & 0 \\
0 & 0 & 0 & c^{2} & 0 & 0
\end{array}\right) \text {, }
$$


where the additional $n G$ in the name of the matrix refers to the fact that the system is not Galilean invariant.

In the following sections, we introduce Roe-type discretizations for these systems and test them using two prototypical cases.

\section{Roe matrices for SMHD}

In order to arrive at numerical schemes with a high resolution of physical phenomena and a low impact of divergence errors on the solution, it is advantageous to apply Roe-type schemes since they allow us to adjust the numerical viscosity for all waves separately. The first steps involve finding Roe-linearizations for above systems, which is not a trivial task since most of the systems are not even conservative.

\subsection{Roe mean values and Roe matrix for the standard equations}

In [35], Roe introduces the concept of consistent local linearizations. If we consider a Riemann problem with left and right states $\boldsymbol{q}_{l}$ and $\boldsymbol{q}_{r}$ and let $\boldsymbol{f}(\boldsymbol{q})$ denote the conservative flux and $\boldsymbol{A}(\boldsymbol{q})$ the corresponding flux Jacobian, then $\tilde{A}\left(\boldsymbol{q}_{l}, \boldsymbol{q}_{r}\right)$ is called a Roe matrix, i. e. a matrix which defines a consistent local linearization for the given Riemann data, if it satisfies

$$
\begin{gathered}
\boldsymbol{f}\left(\boldsymbol{q}_{r}\right)-\boldsymbol{f}\left(\boldsymbol{q}_{l}\right)=\tilde{\boldsymbol{A}}\left(\boldsymbol{q}_{l}, \boldsymbol{q}_{r}\right)\left(\boldsymbol{q}_{r}-\boldsymbol{q}_{l}\right), \\
\tilde{\boldsymbol{A}}\left(\boldsymbol{q}_{l}, \boldsymbol{q}_{r}\right) \rightarrow \boldsymbol{A}(\boldsymbol{q}) \quad \text { for } \quad\left(\boldsymbol{q}_{l}, \boldsymbol{q}_{r}\right) \rightarrow(\boldsymbol{q}, \boldsymbol{q}), \\
\tilde{\boldsymbol{A}}\left(\boldsymbol{q}_{l}, \boldsymbol{q}_{r}\right) \text { is diagonalizable for all } \boldsymbol{q}_{l}, \boldsymbol{q}_{r} .
\end{gathered}
$$

If there exists a single state $\tilde{\boldsymbol{q}}=\tilde{\boldsymbol{q}}\left(\boldsymbol{q}_{l}, \boldsymbol{q}_{r}\right)$ with

$$
\tilde{\boldsymbol{A}}\left(\boldsymbol{q}_{l}, \boldsymbol{q}_{r}\right)=\boldsymbol{A}(\tilde{\boldsymbol{q}}),
$$

then this state is called a Roe mean value for $\boldsymbol{q}_{l}, \boldsymbol{q}_{r}$. For resonant hyperbolic systems, condition (43) has to be relaxed since $\boldsymbol{A}_{\text {standard }}(\boldsymbol{q})$ itself is not always diagonalizable, but still has real eigenvalues. 
For the conservative SMHD system (1) - (3), it is known [36] that $\tilde{\boldsymbol{q}}$ defined by

$$
\begin{gathered}
\tilde{h}=\frac{1}{2}\left(h_{l}+h_{r}\right), \\
\tilde{u}=\frac{\sqrt{h_{l}} u_{l}+\sqrt{h_{r}} u_{r}}{\sqrt{h_{l}}+\sqrt{h_{r}}}, \\
\tilde{v}=\frac{\sqrt{h_{l}} v_{l}+\sqrt{h_{r}} v_{r}}{\sqrt{h_{l}}+\sqrt{h_{r}}}, \\
\tilde{B}_{1}=\frac{\sqrt{h_{l}} B_{1 l}+\sqrt{h_{r}} B_{1 r}}{\sqrt{h_{l}}+\sqrt{h_{r}}}, \\
\tilde{B}_{2}=\frac{\sqrt{h_{l}} B_{2 l}+\sqrt{h_{r}} B_{2 r}}{\sqrt{h_{l}}+\sqrt{h_{r}}}
\end{gathered}
$$

is a Roe mean value, i. e. $\boldsymbol{A}(\tilde{\boldsymbol{q}})$ is a Roe-matrix as discussed above: although not always diagonalizable, it still has real eigenvalues.

If we augment $\tilde{\boldsymbol{q}}$ by an arbitrary mean $\tilde{\psi}$ of $\psi_{l}$ and $\psi_{r}$, then $\boldsymbol{A}_{\mathrm{GLM}}(\tilde{\boldsymbol{q}})$ becomes a Roe matrix for the GLM-system. This becomes clear when considering that $\boldsymbol{A}_{\text {GLM }}$ (cf. equation (18)) does not depend on $\psi$, and the additional non-zero entries compared to $\boldsymbol{A}_{\text {standard }}$ (cf. equation (5)) are mere constants. If $c_{\psi}$ is chosen large enough, it is even guaranteed that $\boldsymbol{A}_{\mathrm{GLM}}(\tilde{\boldsymbol{q}})$ satisfies all conditions for a Roe matrix, including diagonalizability.

\subsection{Roe matrices for Powell with and without GLM}

In order to be able to generalize the Roe scheme to non-conservative systems, one has to replace conditions (41) - (43) by conditions which are independent from the flux function. As Toumi [42] pointed out, it is sufficient to replace (41). It is well known that (for sufficiently smooth $f$ )

$$
\boldsymbol{f}\left(\boldsymbol{q}_{r}\right)-\boldsymbol{f}\left(\boldsymbol{q}_{l}\right)=\int_{0}^{1} A(\phi(s)) \phi_{s}(s) \mathrm{d} s
$$

for any path $\phi:[0,1] \rightarrow \mathbb{R}^{d}$ with $\phi(0)=\boldsymbol{q}_{l}, \phi(1)=\boldsymbol{q}_{r}$, and $d$ being the dimension of the state space. Thus, a suitable replacement for (41) has to take on the form

$$
\int_{0}^{1} A(\phi(s)) \phi_{s}(s) \mathrm{d} s=\tilde{\boldsymbol{A}}\left(\boldsymbol{q}_{l}, \boldsymbol{q}_{r}\right)\left(\boldsymbol{q}_{r}-\boldsymbol{q}_{l}\right) .
$$

The main difference between the conservative and the non-conservative case is that in the latter different choices of $\phi$ might lead to different Roe matrices.

Considering Toumi [42], such a Roe matrix can be obtained by generalizing the approach in [35]. For given left and right states $\boldsymbol{q}_{l}$ and $\boldsymbol{q}_{r}$, parametrize $\boldsymbol{q}$ as $\boldsymbol{q}=\boldsymbol{q}\left(\boldsymbol{w}_{l}+(1-s) \boldsymbol{w}_{r}\right)$ 
with $\boldsymbol{q}\left(\boldsymbol{w}_{l}\right)=\boldsymbol{q}_{l}$ and $\boldsymbol{q}\left(\boldsymbol{w}_{r}\right)=\boldsymbol{q}_{r}$. Thus, $\boldsymbol{\phi}$ becomes $\phi(s)=\boldsymbol{q}\left(\boldsymbol{w}_{l}+(1-s) \boldsymbol{w}_{r}\right)$. With that, the integral on the left-hand side of equation (47) becomes

$$
\int_{0}^{1} A(\phi(s)) \phi_{s}(s) \mathrm{d} s=\int_{0}^{1} \boldsymbol{A}\left(\boldsymbol{q}\left(\boldsymbol{w}_{l}+(1-s) \boldsymbol{w}_{r}\right)\right) \cdot \boldsymbol{A}_{0}\left(\boldsymbol{w}_{l}+(1-s) \boldsymbol{w}_{r}\right) \mathrm{d} s \cdot\left(\boldsymbol{w}_{r}-\boldsymbol{w}_{l}\right),
$$

where $\boldsymbol{A}_{0}$ is the Jacobian of $\boldsymbol{q}$ with respect to $\boldsymbol{w}$. On the other hand, for $\boldsymbol{A}_{0}$ the mean value theorem implies

$$
\boldsymbol{q}_{r}-\boldsymbol{q}_{l}=\int_{0}^{1} \boldsymbol{A}_{0}\left(s \boldsymbol{w}_{r}+(1-s) \boldsymbol{w}_{l}\right) \mathrm{d} s \cdot\left(\boldsymbol{w}_{r}-\boldsymbol{w}_{l}\right) .
$$

The integral on the right-hand side of equation (49) is non-singular for any reasonable parametrization of $\boldsymbol{q}$, thus

$$
\tilde{\boldsymbol{A}}\left(\boldsymbol{q}_{l}, \boldsymbol{q}_{r}\right)=\int_{0}^{1} \boldsymbol{A}\left(\boldsymbol{q}\left(s \boldsymbol{w}_{l}+(1-s) \boldsymbol{w}_{r}\right)\right) \cdot \boldsymbol{A}_{0}\left(\boldsymbol{w}_{r}+(1-s) \boldsymbol{w}_{l}\right) \mathrm{d} s \cdot\left(\int_{0}^{1} \boldsymbol{A}_{0}\left(\boldsymbol{w}_{l}+(1-s) \boldsymbol{w}_{r}\right) \mathrm{d} s\right)^{-1}
$$

is a Roe matrix with respect to the path $\phi(s)=\boldsymbol{q}\left(s \boldsymbol{w}_{r}+(1-s) \boldsymbol{w}_{l}\right)$.

For the standard SMHD system (1) - (3) the usual choice is

$$
\boldsymbol{w}:=\left(\sqrt{h}, \sqrt{h} u, \sqrt{h} v, \sqrt{h} B_{1}, \sqrt{h} B_{2}\right)^{T}
$$

and, thus,

$$
\boldsymbol{q}(\boldsymbol{w})=\sqrt{h} \boldsymbol{w} .
$$

This leads to the Roe matrix given in [36], which is just the matrix $\boldsymbol{A}(\tilde{\boldsymbol{q}})$ described in the previous section.

If the fourth column in the system matrix $\boldsymbol{A}_{\text {Powell }}$ for the system with the Powell correction (equation (26) ) had only the diagonal entry, the Roe matrix given our choice for $\boldsymbol{w}$ would simply be the matrix $\boldsymbol{A}_{\text {Powell }}(\tilde{\boldsymbol{q}})$. But the entry in the second row causes significant changes in the first column of the Roe matrix:

$$
A_{1}=\left(\begin{array}{ccccc}
0 & 1 & 0 & 0 & 0 \\
-\tilde{u}^{2}+\frac{1}{2}\left[\tilde{B}_{1}^{2}+\widehat{B_{1}^{2}}\right]+g \tilde{h} & 2 \tilde{u} & 0 & -\tilde{B}_{1} & 0 \\
-\tilde{u} \tilde{v}+\frac{1}{2}\left[\tilde{B}_{1} \tilde{B}_{2}+\widehat{B_{1} B_{2}}\right] & \tilde{v} & \tilde{u} & 0 & -\tilde{B}_{1} \\
\frac{1}{2}\left[\widehat{u B_{1}}-\tilde{u} \tilde{B}_{1}\right] & 0 & 0 & \tilde{u} & 0 \\
\frac{1}{2}\left[\tilde{v} \tilde{B}_{1}+\widehat{v B_{1}}\right]-\tilde{u} \tilde{B}_{2} & \tilde{B}_{2} & -\tilde{B}_{1} & 0 & \tilde{u}
\end{array}\right),
$$

where, e. g., the hatted term in the last row is defined as

$$
\widehat{v B_{1}}=\frac{\overline{\left(\frac{w_{3} w_{4}}{w_{1}}\right)}}{\bar{w}_{1}}
$$


with

$$
\overline{\left(\frac{w_{3} w_{4}}{w_{1}}\right)}:=\int_{0}^{1} \frac{\left(s w_{3 r}+(1-s) w_{3 l}\right)\left(s w_{4 r}+(1-s) w_{4 l}\right)}{\left(s w_{1 r}+(1-s) w_{1 l}\right)} \mathrm{d} s
$$

and

$$
\bar{w}_{1}:=\int_{0}^{1} s w_{1 r}+(1-s) w_{1 l} \mathrm{~d} s=\frac{w_{1 r}+w_{1 l}}{2},
$$

where the $w_{k}$ are the components of the parameter vector $\boldsymbol{w}$ as defined in equation (50). (The other hatted terms are defined likewise.)The integral in equation (54) can be solved explicitly, but the solution involves many terms, some of them logarithmic. This makes the computation rather time consuming. Furthermore, the fill-in in the fourth row of the first column makes the computation of the eigensystem rather awkward and even more time consuming. Thus, we decide to drop this approach.

In order to avoid unphysical solutions, we have to recall the derivation of the system. As long as there is no magnetic charge or magnetic current, both the conservative standard system and the Galilean invariant Powell system yield the same analytical solutions. Thus, it seems sufficient to extend the Roe matrix for the conservative system in the same way as we did for the analytic case. For our numerical computations, we employ the local linearizations $\boldsymbol{A}_{\text {Powell }}(\tilde{\boldsymbol{q}})$ (equation (26)) and $\boldsymbol{A}_{\mathrm{PGLM}}(\tilde{\boldsymbol{q}})$ (equation (33)) for the Powell case and the Powell-GLM case respectively. This ensures that in the absence of magnetic chargeswhich are just numerical artifacts-we obtain physical solutions. By the construction of the Powell and the Powell-GLM systems, we expect these numerical artifacts to be transported and/or radiated away from the computational domain.

In more detail: If we write the system matrix $\boldsymbol{A}_{\text {Powell }}(\boldsymbol{q})$ of the Powell system as

$$
\boldsymbol{A}_{\text {Powell }}(\boldsymbol{q})=\boldsymbol{A}_{\text {standard }}(\boldsymbol{q})+\boldsymbol{A}_{\text {corr }}(\boldsymbol{q})
$$

and perform the above process for both parts separately, we obtain

$$
\tilde{\boldsymbol{A}}_{\text {Powell }}\left(\boldsymbol{q}_{l}, \boldsymbol{q}_{r}\right)=\boldsymbol{A}_{\text {standard }}(\tilde{\boldsymbol{q}})+\tilde{\boldsymbol{A}}_{\text {corr }}\left(\boldsymbol{q}_{l}, \boldsymbol{q}_{r}\right)
$$

with

$$
\tilde{\boldsymbol{A}}_{\text {corr }}\left(\boldsymbol{q}_{l}, \boldsymbol{q}_{r}\right)=\boldsymbol{A}_{\text {corr }}(\tilde{\boldsymbol{q}})+\left(\begin{array}{ccccc}
0 & 0 & 0 & 0 & 0 \\
\frac{1}{2}\left(\widehat{B_{1}^{2}}-\tilde{B}_{1}^{2}\right) & 0 & 0 & 0 & 0 \\
\frac{1}{2}\left(\widehat{B_{1} B_{2}}-\tilde{B}_{1} \tilde{B}_{2}\right) & 0 & 0 & 0 & 0 \\
\frac{1}{2}\left(\widehat{u B_{1}}-\tilde{u} \tilde{B}_{1}\right) & 0 & 0 & 0 & 0 \\
\frac{1}{2}\left(\widehat{B_{1} B_{2}}-\tilde{v} \tilde{B}_{1}\right) & 0 & 0 & 0 & 0
\end{array}\right) .
$$

The only remaining terms in the second matrix on the right-hand side result from the different averaging strategies. 


\section{Numerical tests}

All tests are done with clawpack [5], an implementation of the wave propagation method, which is based on fluctuation splitting. Thus, it can handle non-conservative systems in a natural way. Since in practice, most computations are done with second or even higher order, we present results only for second order. As limiters, we employ Superpower [18] on the magnetogravitational waves and CFL-superbee on all other waves. The resonant wave in the standard model is computed with first order. The implementation follows [18] and [19], which explain how to apply the Jeng-Payne correction for spatial varying CFLnumbers to arbitrary TVD-limiters.

To avoid expansion shocks, we employ the Harten entropy fix $4[16]$. For this, a parameter $\delta>0$ has to be chosen. For values smaller than $\delta$, the numerical viscosity on the wave is modified and, finally, bounded from below by $\delta / 2$ according to

$$
\phi(\lambda)= \begin{cases}|\tilde{\lambda}| & \text { if }|\tilde{\lambda}| \geq \delta \\ \left(\tilde{\lambda}^{2}+\delta^{2}\right) /(2 \delta) & \text { if }|\tilde{\lambda}|<\delta\end{cases}
$$

where $\phi(\tilde{\lambda})$ denotes the viscosity on the wave corresponding to the eigenvalue $\tilde{\lambda}$ of the Roe matrix. If not stated otherwise, we use $\delta=10^{-8}$ on all waves. For the standard model, we employ as the Harten parameter for the resonant wave

$$
\delta_{\text {resonant }}=2 \max \left\{\left|\tilde{u}-\tilde{c}_{g}\right|,\left|\tilde{u}+\tilde{c}_{g}\right|\right\}=: 2 s_{\max }
$$

as suggested in $[20,21]$. Note that for the standard GLM (cf. equation (18)) and the system determined by equation (40), the application of the Harten entropy fix on the artificial waves is pointless. Their speed is always $c_{\psi}$. Furthermore, $c_{\psi}$ is chosen to be the highest wave speed and, thus, determines the time step. Therefore, raising the viscosity on these waves would give no advantage over choosing the corresponding higher wave speed for $c_{\psi}$. Note that wherever $\phi(\tilde{\lambda}) \neq|\tilde{\lambda}|$, the accuracy of the scheme drops to first order. This is due to the algebraic limiting used in clawpack. As we already pointed out in [21], algebraic limiting is formulated as a correction to a fixed first order scheme, in clawpack the wave-wise standard upwind scheme. If the underlying first order scheme is changed, the correction, in general, cannot lead to second order of the resulting scheme.

The artificial speed $c_{\psi}$ is stated as multiples of $s_{\max }$, which, as indicated in equation (60), represents the highest physical wave speed in the problem. This is done for each time step separately, i. e., before the time step is performed, $c_{\psi}$ is set according to the wave speeds occurring in the last time step.

In order to assess above methods, we consider the normalized $\mathscr{L}^{\infty}-, \mathscr{L}^{2}$-, $\mathscr{L}^{1}$-norms. We also show some pictures of the height and the distribution of the divergence errors to illustrate the effects detected in the plots of the norms. We employ two prototypical test

\footnotetext{
${ }^{4}$ Not to be confused with the Harten-Hyman entropy fix [17]
} 

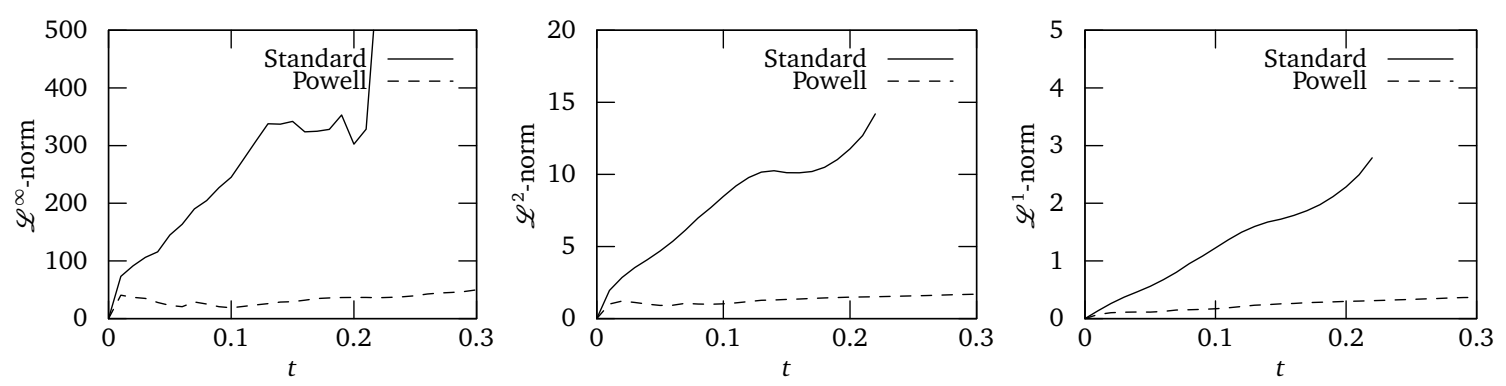

Figure 1: Generalized dam break: Different norms for divergence when computed without GLM.

cases: (1) a generalized radial dam break as an example, where plasma will remain inside the boundary of the computational domain for a long time. This is a challenge especially for the pure Powell correction. (2) the de Sterck test as an example with a high flow speed in $x$-direction. This is a challenge especially for the Powell-GLM system since it might happen that $u-c$ is positive or even vanishes, i. e., the divergence errors could stay in the computational domain for a prolonged time.

\subsection{Generalized radial dam break}

We start with the generalized dam break problem, which was introduced as a test case by Quamar et el. [34]. The initial state is a situation at rest. In addition, $h \boldsymbol{B}$ is constant all over the computational domain and, thus, divergence-free. In the center of the computational domain is a cylindrical region of radius 0.1 with increased height. The initial data for the fluids in the circular and outside regions are:

$$
\begin{aligned}
& h_{\text {in }}=10, \quad u_{\text {in }}=0, \quad v_{\text {in }}=0, \quad B_{1 \text { in }}=0.1, \quad B_{2 \text { in }}=0 \text {, } \\
& h_{\text {out }}=1, \quad u_{\text {out }}=0, \quad v_{\text {out }}=0, \quad B_{1 \text { out }}=1, \quad B_{2 \text { out }}=0 \text {, }
\end{aligned}
$$

and the gravitational constant is normalized to $g=1$. For the consideration of the error norms, we compute up to $t=0.3$. The computational domain is a square region $[-1,1] \times$ $[-1,1]$ with extrapolation boundaries. The domain is discretized with $300 \times 300$ grid cells.

For this generalized radial dam-break experiment, we first consider the results obtained without GLM. Both the standard and the Powell system have in common that all magnetic monopoles stay in the computational domain. For the standard equations since there is no transport of magnetic monopoles at all, for the Powell system since the flow velocity at the boundary is zero. Thus, one would expect the $\mathscr{L}^{1}$-norm of the divergence errors to be identical for both calculations. But, as Figure 1 shows, for the standard system, it is considerably higher. This confirms the major role played by resonance in the production of divergence errors [20]. Although we imposed a high viscosity on the resonant wave, the production of magnetic charges is much higher than for the non-resonant Powell system. Figure 2 shows the solution of both computations shortly before the breakdown of the 

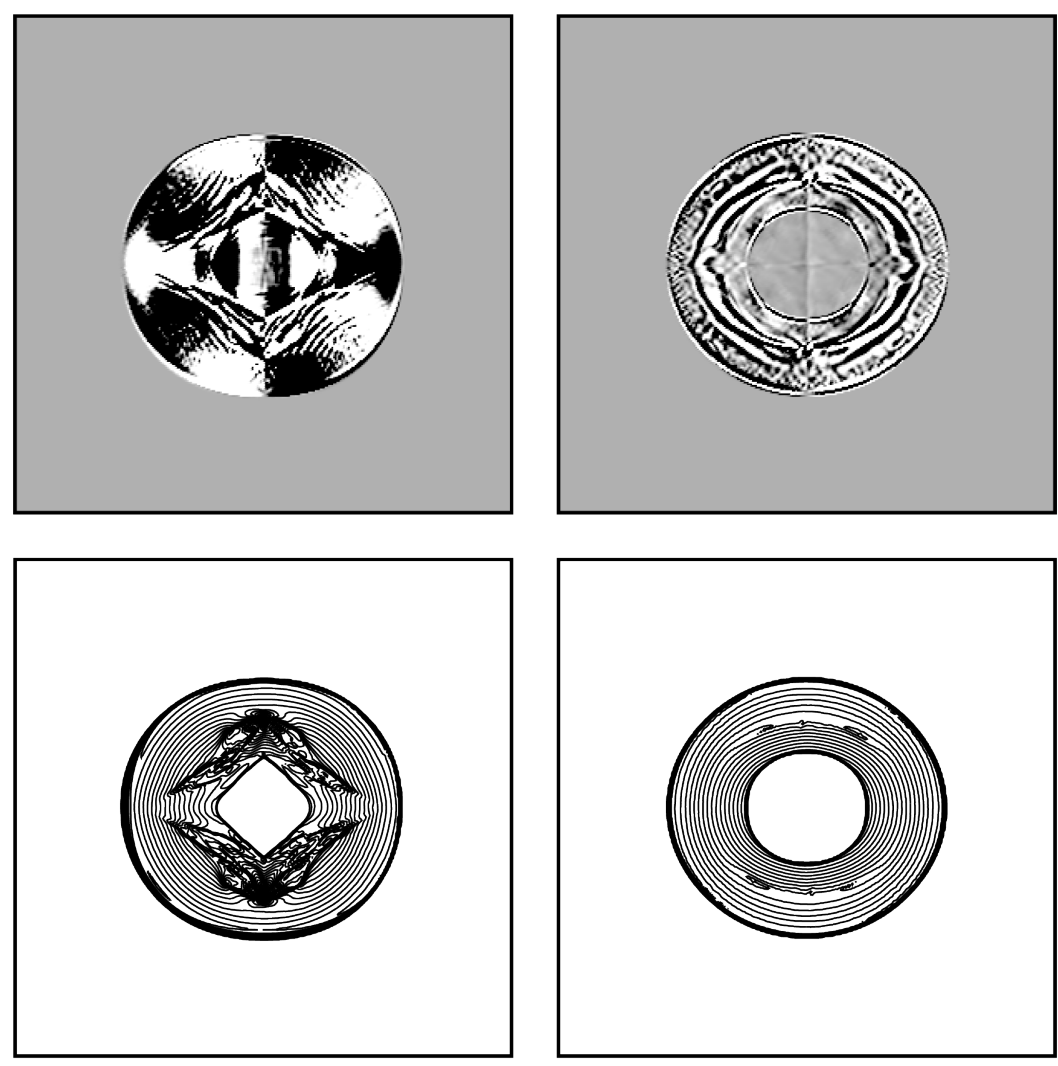

Figure 2: Generalized dam break without GLM at $t=0.18$ : Schlieren type plots for divergence error (upper row) and contour plots for height (lower row); left without divergence correction, right with Powell correction.

standard computation due to excessive divergence errors. For the Schlieren type plots, we employed \pm 0.3 as a threshold for black and white. It is clearly seen that, while for the Powell system the height seems reasonable, the results for the standard system are highly unphysical and thus worthless. It can also be observed that in both cases no magnetic monopoles are found outside of the area confined by the shock.

In Figures 3 and 4, we show different tests for the Powell-GLM system. All results for that system differ from the standard and the Powell computations in one crucial point: after some time all error norms decrease. However, this is impossible for the Powell and the standard system. Because of the initial conditions and the boundary conditions, divergence errors cannot be removed from the computational domain, not even for the Powell system.

In Figure 3, we compare different choices for the viscosity on the artificial waves. All tests are done with the artificial speed $c_{\psi}$ set to $c_{\psi}=2 s_{\max }$. The viscosity is adjusted via the parameter $\delta$ in the Harten entropy fix. Although the implementation of the viscosity as a split of a single wave into two waves running at different speeds formally would require a reduction of the time step size, we computed it using the wave speeds of the original waves. This means that the computational cost is identical for all tested viscosities. As Einfeldt [10] 

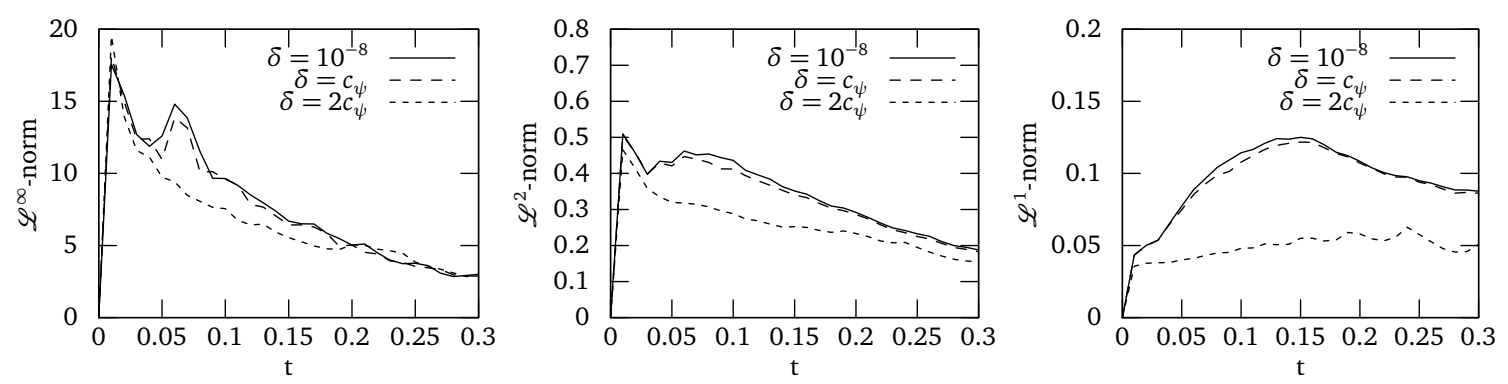

Figure 3: Generalized dam break: Powell + GLM: Comparison for different viscosities; all cases computed with $c_{\psi}=2 s_{\max }$.
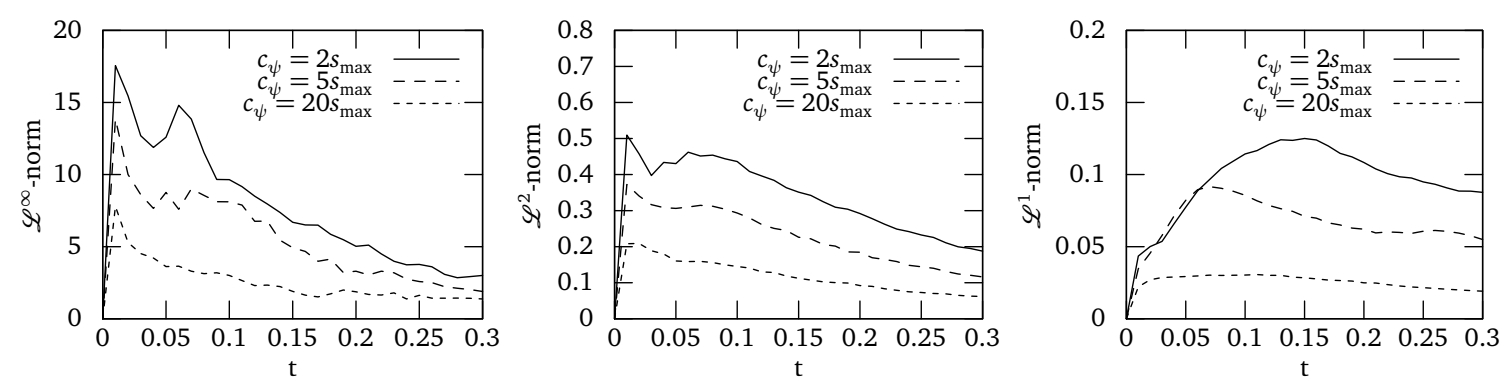

Figure 4: Generalized dam break: Powell + GLM: Comparison for different wave speeds; all cases computed with $\delta=10^{-8}$.

points out, the stability of the scheme does not depend on the faster of those split waves, but on the resulting viscosity superimposed on the original wave. As Figure 3 indicates, the computation is stable even with $\delta=2 c_{\psi}$. This can be inferred from equation (59), which implies that the difference between $|\tilde{\lambda}|$ and $\phi(\tilde{\lambda})$ decreases for increasing $|\tilde{\lambda}|$. Since, for every $t>0$, the flow field contains supercritical regions, we can expect (but, unfortunately, not guarantee) that the maximal value for $|\tilde{u}|$ is larger than $s_{\max } / 2$. Together with the setting $c_{\psi}=2 s_{\max }$, this means that the maximal value for $\left|\tilde{u} \pm c_{\psi}\right|$ (which determines the time step size) is larger than $\frac{5}{4} c_{\psi}$. If we insert this and $\delta=2 c_{\psi}$ in equation (59), we obtain as an upper bound for the viscosity

$$
\phi\left(\frac{5}{4} c_{\psi}\right)=\frac{5}{4} c_{\psi}+\frac{9}{64} c_{\psi}
$$

Since the time step size in our computations is computed with CFL-number 0.9 with respect to the maximal value of $\left|\tilde{u} \pm c_{\psi}\right|$, this implies for the CFL-number with respect to the viscosity an upper bound of 1.0125. From our considerations, it is clear that we usually cannot expect this upper bound to be attained at any time $t>0$. In the initial state of the generalized dam break problem, no supercritical regions are found, thus the above assumptions are not true at $t=0$, and the real CFL-number might be larger than one. This, in turn, might be the reason for the rather high errors observed for the early time steps. 
Note that, since the maximal value for $\left|\tilde{u} \pm c_{\psi}\right|$ is bounded below by $c_{\psi}, \delta=c_{\psi}$ can always be chosen without any restrictions.

The most significant feature in Figure 3 is that the gain in quality obtained by a higher viscosity on the divergence waves is mostly seen for the $\mathscr{L}^{1}$-norm. The differences for the $\mathscr{L}^{\infty}$ - and the $\mathscr{L}^{2}$-norm are small. This indicates that the latter mainly represent the production of the divergence errors at the shock. From that, we conclude that even without resonance, the viscosity on the divergence waves plays an important role.

In Figure 4 we show a comparison for different choices of the artificial wave speed. The parameter $\delta$ is set to its standard value $\delta=10^{-8}$. Here, a difference can be clearly seen. But one has to keep in mind that the better results are at the price of a significantly reduced time step. Choosing $c_{\psi}=20 s_{\max }$ instead of $c_{\psi}=2 s_{\max }$ would reduce the divergence errors by a factor of (roughly) 3 , but might reduce the time step by a factor of up to 10 . As the comparison to Figure 3 shows, for $c_{\psi}=2 s_{\max }, \delta=2 c_{\psi}$ yields results of a quality almost comparable to the results for $c_{\psi}=5 s_{\max }, \delta=10^{-8}$. While the latter is better in terms of the $\mathscr{L}^{\infty}$-norm, the previous results in a lower $\mathscr{L}^{1}$-norm. The $\mathscr{L}^{2}$-norm is about the same in both cases. This confirms that in general it is sufficient to choose $c_{\psi}=2 s_{\max }, \delta=2 c_{\psi}$, which allows a higher time step size than $c_{\psi}=5 s_{\max }, \delta=10^{-8}$.

Figure 5 shows the results for $c_{\psi}=2 s_{\max }, \delta=2 c_{\psi}$ and $c_{\psi}=20 s_{\max }, \delta=10^{-8}$. The thresholds for the Schlieren type plots of the divergence errors are the same as in Figure 2 The divergence errors are concentrated at the discontinuities, i. e. just where they are produced. Looking at it in more detail, one finds that even outside the area confined by the shock, some marbled structures are seen in the divergence plots. This indicates, that the divergence errors are transported to the outside. Although the divergence errors are higher for $c_{\psi}=2 s_{\max }, \delta=2 c_{\psi}$, they are considerably better than those without GLM (cf. Figure 2 ). The contour plots for the height are almost indistinguishable for both sets of the parameters. Thus, we conclude that in general it is sufficient to choose $c_{\psi}=2 s_{\max }, \delta=2 c_{\psi}$. The slight loss in quality compared to $c_{\psi}=20 s_{\max }, \delta=10^{-8}$ is outweighed by the considerable saving in computation time.

In Figure 6, we present the error norms for different choices of $c_{\psi}$ when used within the conservative (and, thus, non-Galilean-invariant) GLM-system. Remember that in this case the application of the Harten entropy fix on the artificial waves is pointless since their wave speed is constant in space and determines the time step. A comparison with Figure 4 reveals that the Galilean invariant (and, thus, non-conservative) GLM-system is slightly better behaved, at least for this test case. With respect to the higher computational effort for the eigensystem of the conservative GLM-system, this is an encouraging result.

Finally, Figure 7 shows that the system defined in equation (40) is not able to provide any enhancement. Only the standard equations lead to poorer results.

\subsection{The de Sterck test}

The De Sterck test [39] is a special configuration for a shallow water MHD flow. It shows a strong tendency to develop resonant phenomena and, thus, to single out numerical 

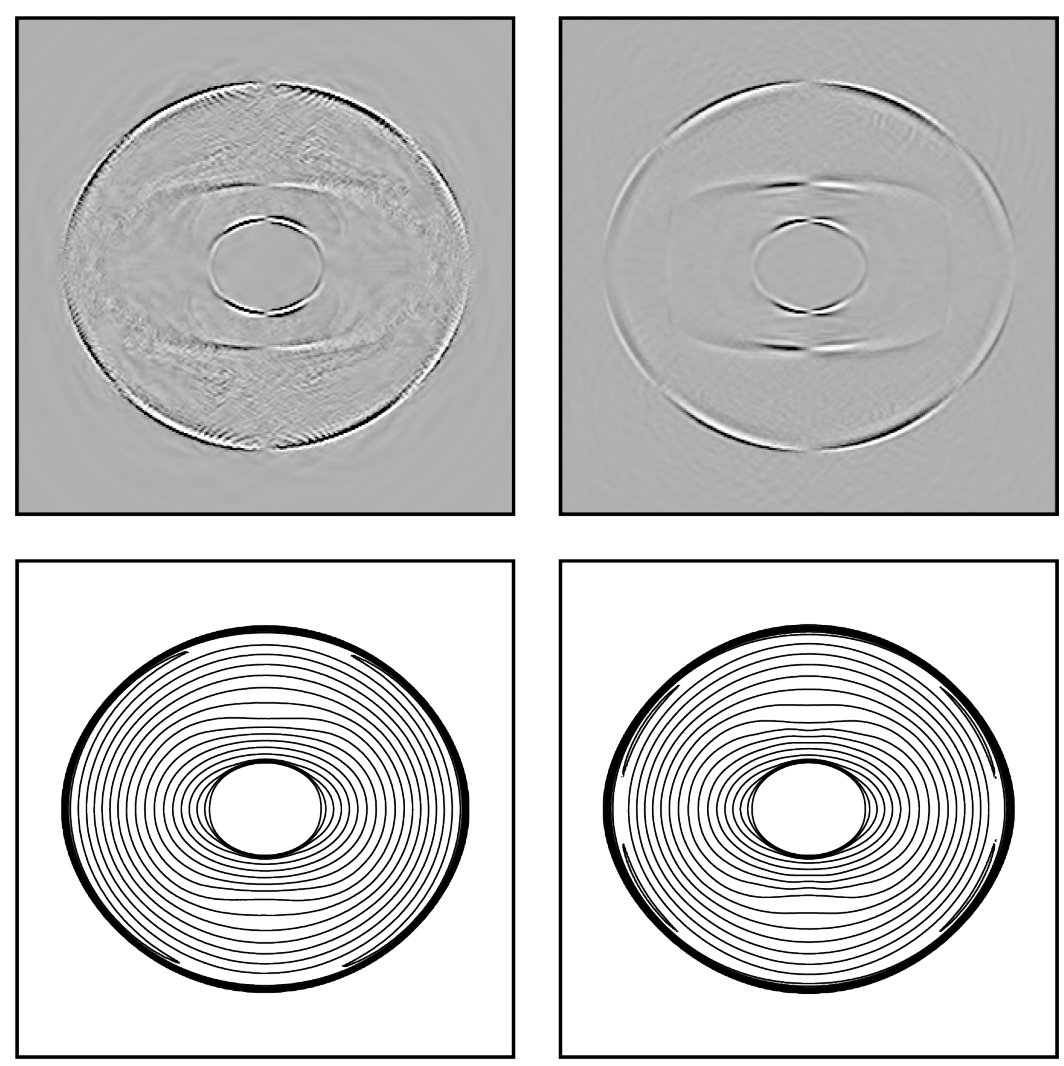

Figure 5: Generalized dam break with Powell+GLM at $t=0.3$ : Schlieren type plots for divergence error (upper row) and contour plots for height (lower row); left with $c_{\psi}=2 s_{\max }, \delta=2 c_{\psi}$, right with $c_{\psi}=20 s_{\max }, \delta=10^{-8}$.
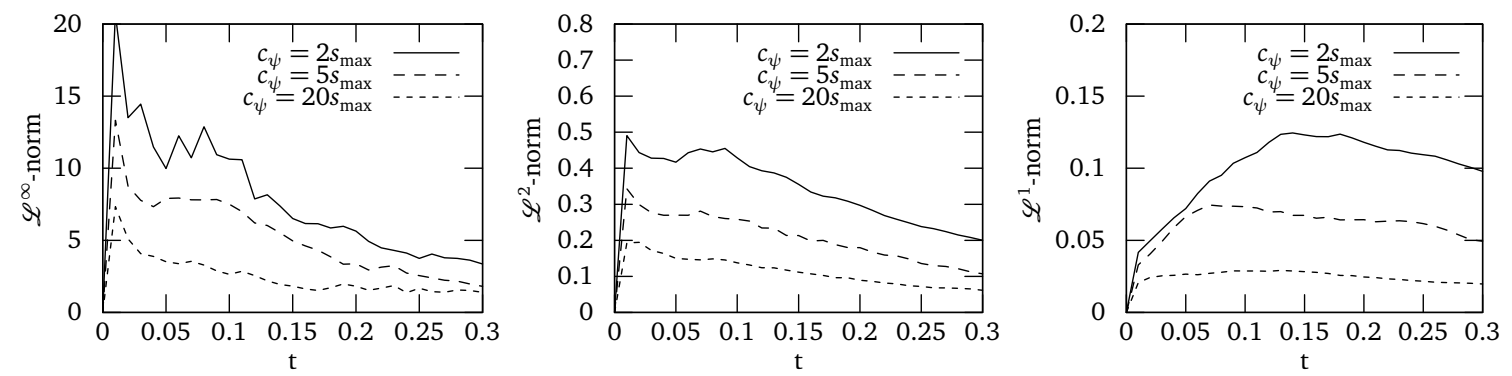

Figure 6: Generalized dam break with standard GLM: Comparison for different wave speeds; all cases computed with $\delta=10^{-8}$. 

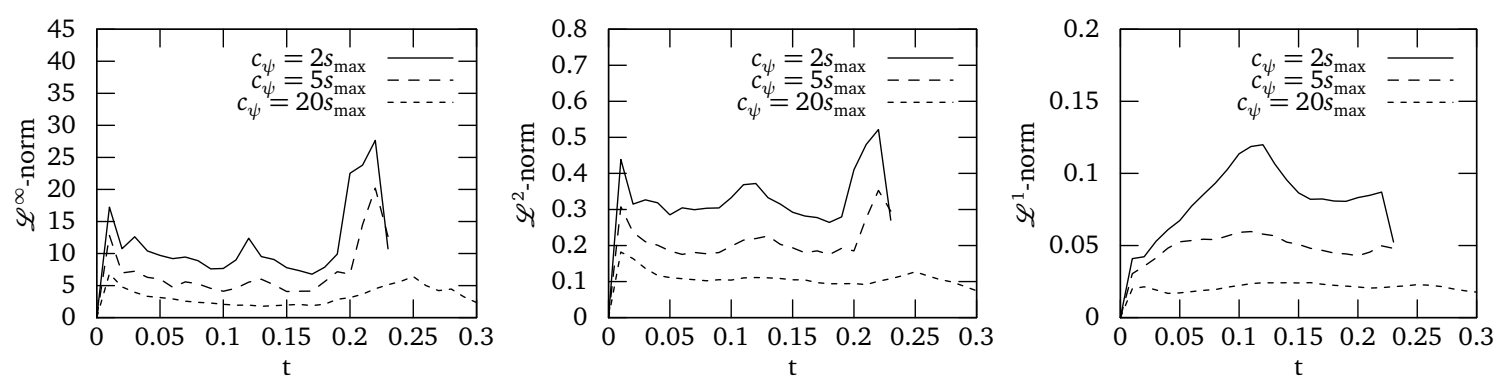

Figure 7: Generalized dam break: Non Galilean invariant, note the changed scaling for the $\mathscr{L}^{\infty}$-norm.
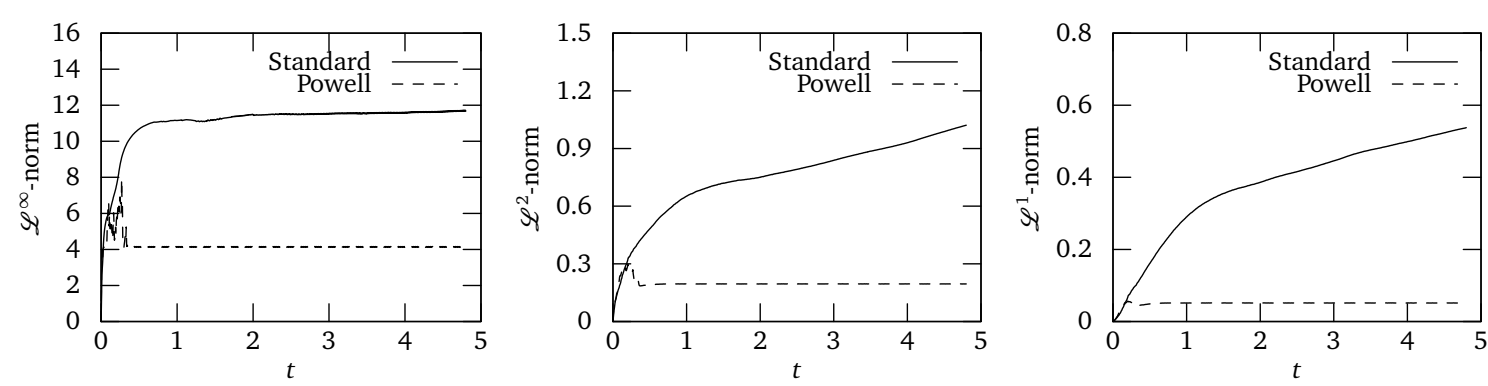

Figure 8: Error norms for de Sterck test without GLM.

schemes which are prone to divergence errors. The test problem imposes a supercritical, horizontal grid-aligned inflow on the left boundary of a rectangular domain. The initial state in the lower half of the domain, and also the boundary conditions at the left boundary, contain a resonant mode. The initial data in the upper half are

$$
h=2, \quad u=5.5, \quad v=0, \quad B_{1}=0.5, \quad B_{2}=0,
$$

and in the lower half

$$
h=1, \quad u=4.5, \quad v=0, \quad B_{1}=2, \quad B_{2}=0 .
$$

The gravitational constant is set to one. Since the discontinuity is aligned with the grid, the initial data are discrete divergence free for any reasonable difference operator. We performed our tests on a $100 \times 100$ grid for the domain $[-1,1] \times[-1,1]$. We compute up to time $t=4.8$, which is about six times the duration it would take to arrive-physically-at a steady state.

Figure 8 shows the error norms for the standard equations and for the Powell system. They indicate that for the Powell system the divergence errors become stationary fairly soon, which in turn means that the Powell system is suitable for steady state computations. Without any divergence correction, the maximum of the divergence errors is almost stationary. But as the other two norms indicate, divergence errors of lower amplitude are produced during the whole computation. The fact that the growth rate of the $\mathscr{L}^{2}$-norm 

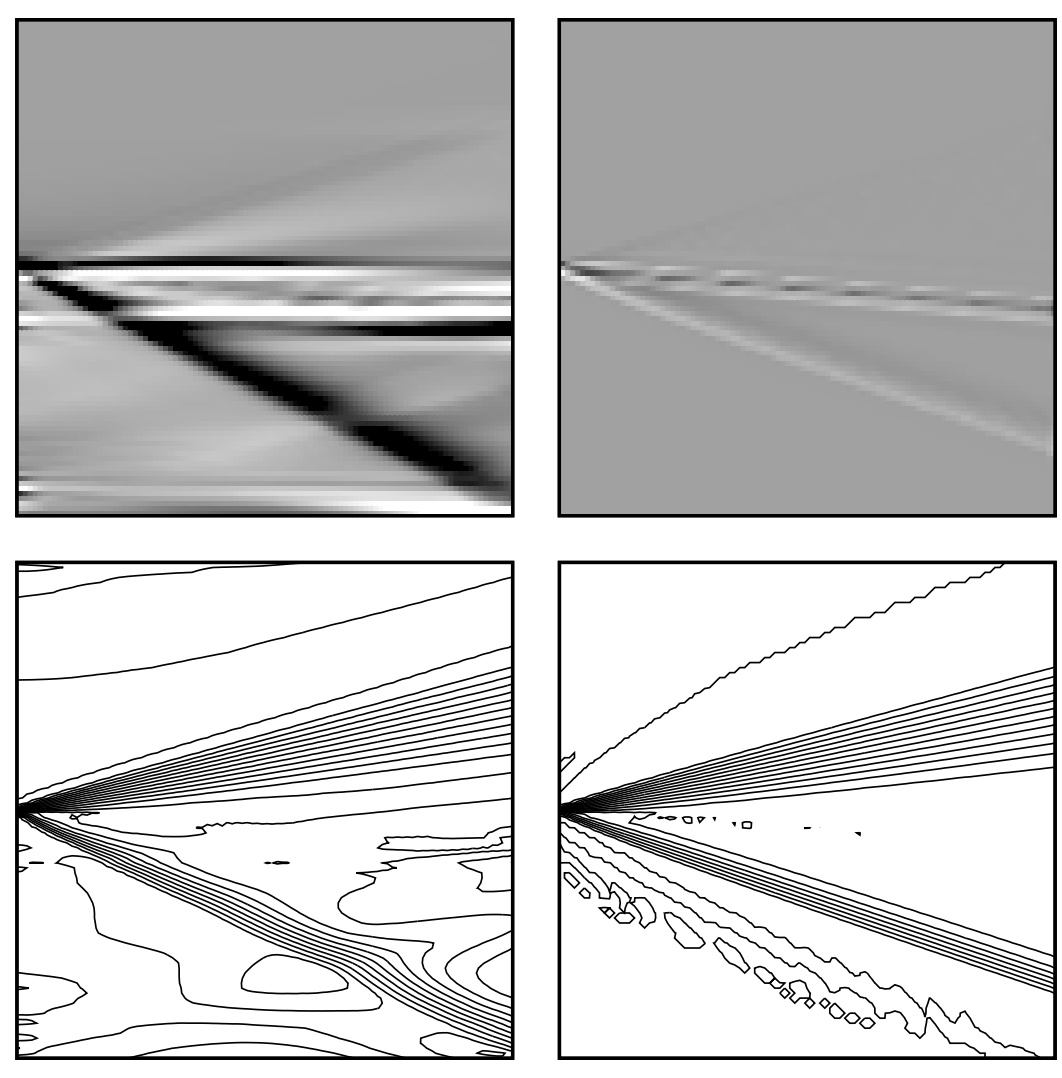

Figure 9: De Sterck test without GLM at $t=4.8$ : Schlieren type plots for divergence error (upper row) and contour plots for height (lower row); left without divergence correction, right with Powell correction.

is almost the same as for the $\mathscr{L}^{1}$-norm indicates that the newly generated divergence errors are still of significant size. The effect of the divergence errors on the solution is illustrated in Figure 9. In this case, the threshold for black and white in the divergence plots is set to \pm 2 . While the physical solution is already stationary at $t=0.6$, we continued the computation up to $t=4.8$. Figure 9 indicates that the Powell system yields a good representation of the physical solution even after such a long-term run, while for the standard system a bend in one of the wave fronts is observed.

In Figures 10 and 11, we show comparisons for different settings of viscosity and speed of the artificial waves in the Powell-GLM system. Like for the generalized dam break, the choice $c_{\psi}=2 s_{\max }, \delta=2 c_{\psi}$ results in about the same error norms as $c_{\psi}=s_{\max }, \delta=10^{-8}$. Increasing the wave speed to $c_{\psi}=20 s_{\max }$ only reduces the errors by a factor less than $3 / 2$. In Figure 12, we show the results for the height. The results with $c_{\psi}=2 s_{\max }, \delta=2 c_{\psi}$ are slightly better than for the pure Powell correction (Figure 9). The gain in quality for $c_{\psi}=20 s_{\max }, \delta=10^{-8}$ is however rather small.

For the pure GLM correction, the results are worse than for the combined Powell-GLM system. We had to choose $c_{\psi} \geq 3.7$ to arrive at a stable scheme. For higher values of $c_{\psi}$, 

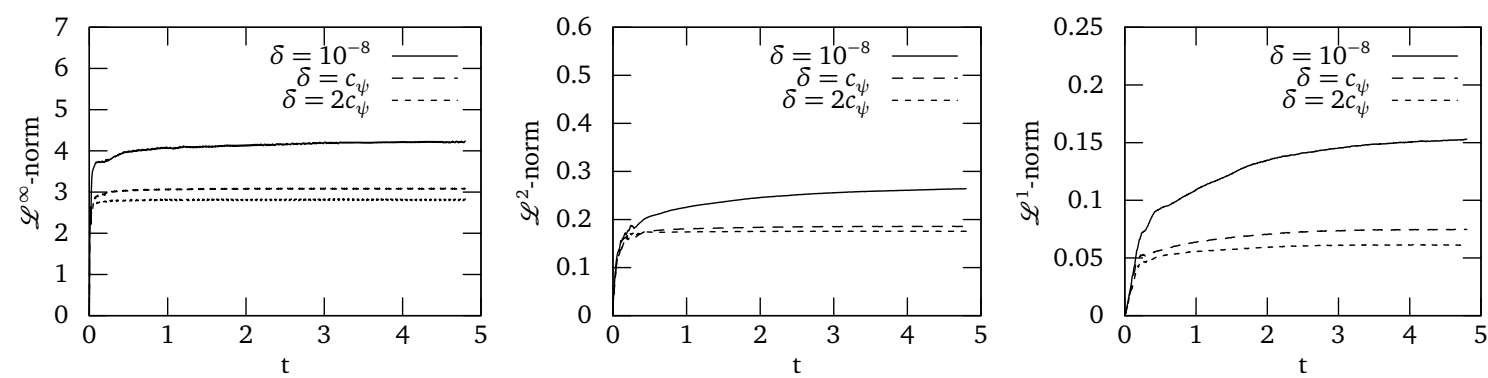

Figure 10: Error norms for de Sterck test with Powell+GLM and $c_{\psi}=2 s_{\max }$ for different viscosities.
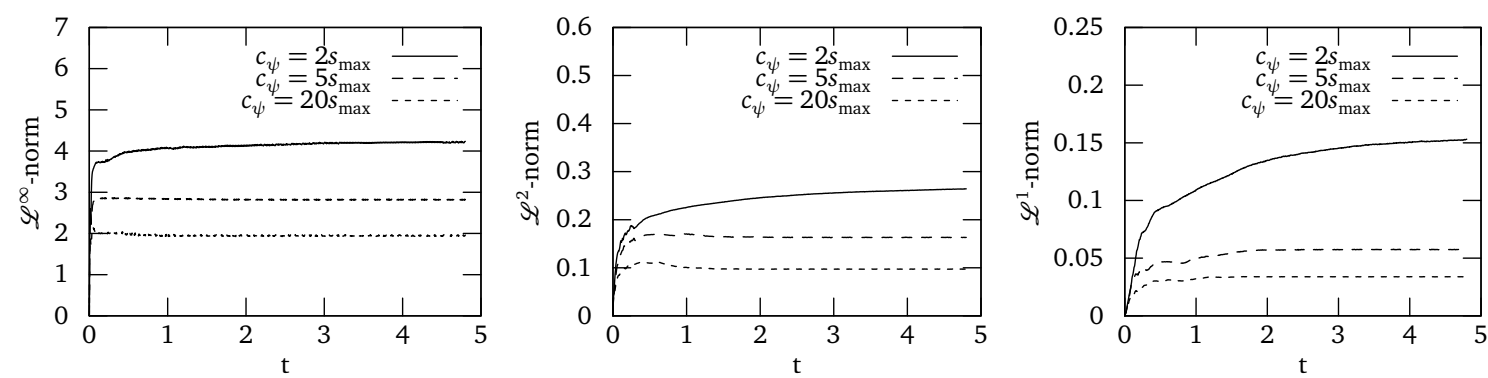

Figure 11: Error norms for de Sterck test with Powell+GLM and different choices for the artificial wave speed.

the errors are still slightly higher than when using Powell and GLM together. Note that all presented results are still considerably improved in comparison to what one could achieve without any divergence correction [20,21]. For the system that combines the eigenvalues of standard GLM and the eigenvectors of Powell GLM (cf. equations (40) and (39)), even with $c_{\psi}=20 s_{\max }$, the computation failed due to excessive divergence errors.

\section{Conclusions}

In this paper, we discussed several forms of the shallow water magnetohydrodynamics equations and their Roe-type discretizations. Two of these models, the standard form and the equations with the standard application of the hyperbolic Generalized Lagrange Multiplier (GLM) correction, are conservation systems. We also discussed two Galilean invariant forms: the Powell system and the Powell system with hyperbolic GLM. A special case is the modification described at the end of Section 2.2.2. It combines the eigenvalues of standard GLM with the eigenvectors of the Galilean invariant GLM system, a strategy which reduces the computational effort for the eigensystem when compared to standard GLM. Unfortunately, this version yields poor numerical results and is, therefore, not suitable for practical application. 

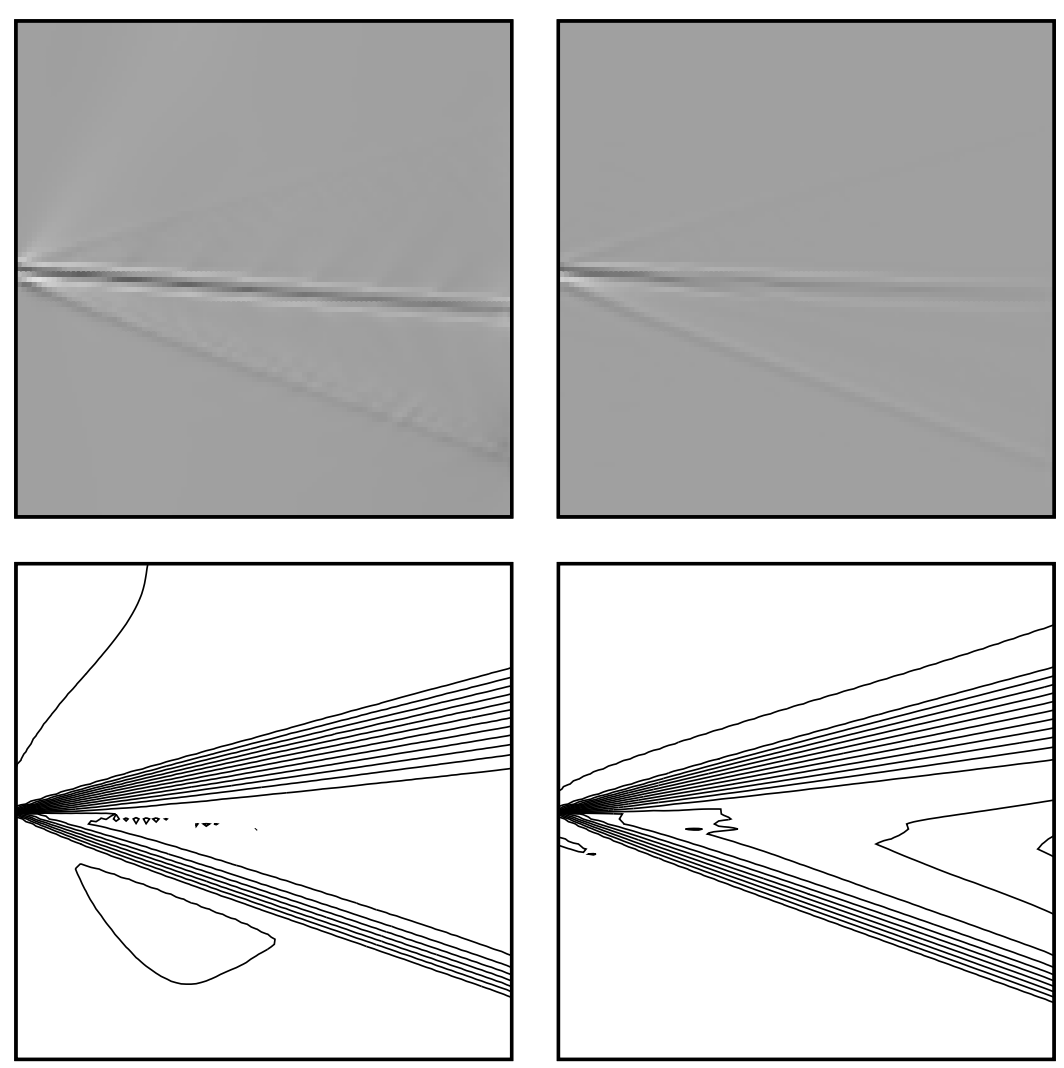

Figure 12: De Sterck test with Powell+GLM at $t=4.8$ : Schlieren type plots for divergence error (upper row) and contour plots for height (lower row); left without divergence correction, right with Powell correction.
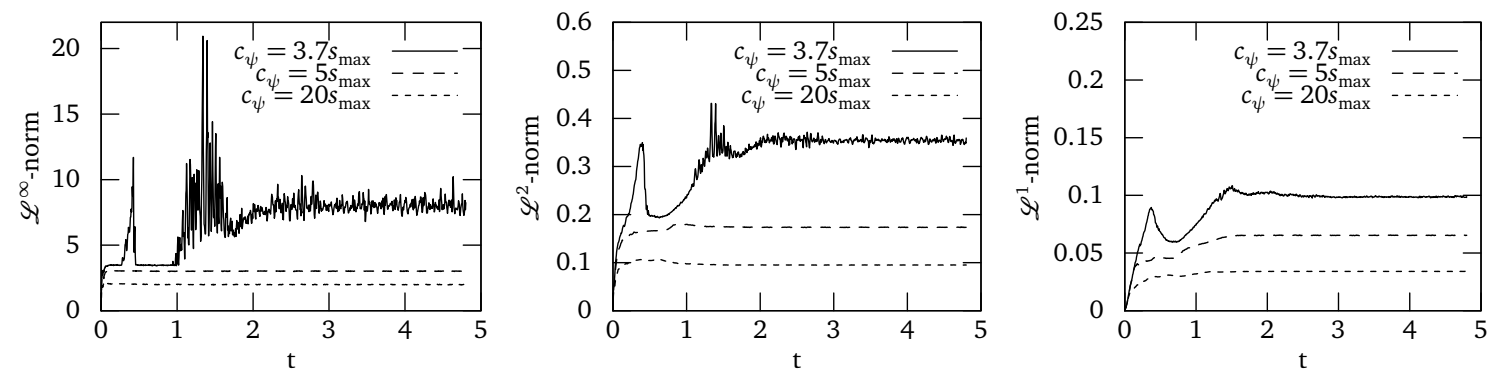

Figure 13: Error norms for de Sterck test with pure GLM and different choices for the artificial wave speed. Note the different scaling for the $\mathscr{L}^{\infty}$-norm. 
As found in Section 2, in the absence of magnetic monopoles, the waves are essentially the same for the conservative ant the non-conservative models. (Remember that all artificial waves vanish in that case.) Thus, the best one can do is to apply a solver based on the characteristic decomposition. Thus, we resorted to Roe-type solvers. Furthermore, the solver for any of the non-conservative models should also coincide with a conservative solver for a solenoidal magnetic field. We showed how the usual way to obtain Roe matrices for the non-conservative systems not only involves computationally expensive logarithms of the physical quantities, but also leads to fill-in in the matrix structure, which in turn results in a highly complicated and computationally even more expensive eigensystem. Without magnetic monopoles, or even artificial monopoles resulting from numerical errors, all physics is represented by the standard equations (1) - (3). We therefore concluded that it is reasonable to resort to the system matrix evaluated in the Roe mean value for the standard equations. The requirement for the solver to coincide with a conservative solver for a solenoidal magnetic field is still satisfied.

Our numerical tests in the last section confirm the superiority of the non-conservative models over the conservative models, especially the standard model. Even the pure the Powell system without additional GLM performs much better than the standard system. As was seen in the case of the generalized dam break problem, this is mainly due to its full hyperbolicity and thus the prevention of any resonant behaviour. In the highly advection dominated De Sterck test, it even performed similar to some versions of the combined Powell+GLM system. From the results in Section 4, we conclude that in such cases, i. e. when the advection allows an efficient transport of the divergence errors out of the computational domain, it is sufficient to employ the Powell system. In terms of computational cost, it is even preferable, since it allows for longer time steps than the GLM versions. But in general, it is favourable to use the combined Powell+GLM system with $c_{\psi}=2 s_{\max }, \delta=2 c_{\psi}$, which is sufficient to provide high quality, physically relevant results.

\section{Acknowledgments}

This work was supported by DFG research grant KE 1420/3-1.

\section{References}

[1] Dinshaw S. Balsara, Divergence-free adaptive mesh refinement for magnetohydrodynamics, J. Comput. Phys. 174 (2001), 614-648.

[2] Dinshaw S. Balsara and Daniel S. Spicer, A staggered mesh algorithm using high order Godunov fluxes to ensure solenoidal magnetic fields in magnetohydrodynamic simulations, J. Comput. Phys. 149 (1999), 270-292.

[3] Nicolas Besse and Dietmar Kröner, Convergence of locally divergence-free discontinuous- 
Galerkin methods for the induction equations of the 2D-MHD system, M2AN Math. Model. Numer. Anal. 39 (2005), no. 6, 1177-1202.

[4] Jeremiah U. Brackbill and D. C. Barnes, The effect of nonzero $\nabla \cdot B$ on the numerical solution of the magnetohydrodynamic equations, J. Comput. Phys. 35 (1980), 426-430.

[5] Clawpack (conservation laws package), http://www.amath.washington.edu/ claw.

[6] Robert K. Crockett, Phillip Colella, Robert T. Fisher, Richard I. Klein, and Christopher F. McKee, An unsplit, cell-centered godunov method for ideal MHD, J. Comput. Phys. 203 (2005), no. 2, 422-448.

[7] Constantine M. Dafermos, Quasilinear hyperbolic systems with involutions, Arch. Ration. Mech. Anal. 94 (1986), 373-389.

[8] _ Hyperbolic conservation laws in continuum physics, Springer, Berlin, Heidelberg, 2000.

[9] Andreas Dedner, Friedemann Kemm, Dietmar Kröner, Claus-Dieter Munz, Thomas Schnitzer, and Matthias Wesenberg, Hyperbolic divergence cleaning for the MHD equations, J. Comput. Phys. 175 (2002), no. 2, 645-673.

[10] Bernd Einfeldt, On Godunov-type methods for gas dynamics., SIAM J. Numer. Anal. 25 (1988), no. 2, 294-318 (English).

[11] C. R. Evans and John F. Hawley, Simulation of general relativistic magnetohydrodynamic flows: A constrained transport method, Astrophys. J. 332 (1988), 659.

[12] F.G. Fuchs, S. Mishra, and N.H. Risebro, Splitting based finite volume schemes for ideal MHD equations., J. Comput. Phys. 228 (2009), no. 3, 641-660 (English).

[13] Franz G. Fuchs, Kenneth H. Karlsen, Siddharta Mishra, and Nils H. Risebro, Stable upwind schemes for the magnetic induction equation., ESAIM, Math. Model. Numer. Anal. 43 (2009), no. 5, 825-852 (English).

[14] Peter A. Gilman, Magnetohydrodynamic "shallow water" equations for the solar tachocline, Astrophys J. Letters 544 (2000), no. 2, L79.

[15] S.K. Godunov, Symmetric form of the magnetohydrodynamic equation, Chislennye Metody Mekh. Sploshnoi Sredy 3 (1972), no. 1, 26-34.

[16] Amiram Harten, High resolution schemes for hyperbolic conservation laws, J. Comput. Phys. 49 (1983), no. 3, 357-393.

[17] Amiram Harten and James M. Hyman, Self adjusting grid methods for one-dimensional hyperbolic conservation laws, J. Comput. Phys. 50 (1983), no. 2, 235-269. 
[18] Friedemann Kemm, A comparative study of TVD limiters-well known limiters and an introduction of new ones, Internat. J. Numer. Methods Fluids 67 (2011), no. 4, 404-440.

[19] _ CFL-number-dependent TVD-limiters, Numerical Methods for Hyperbolic Equations: Theory and Applications (Elena Vázquez-Cendón, Arturo Hidalgo, Pilar García-Navarro, and Luis Cea, eds.), CRC Press, 2012, Proceedings of the international conference on Numerical Methods for Hyperbolic Equations: Theory and Applications, Santiago de Compostela, Spain, 4-9 July 2011, pp. 277-283.

[20] - On the origin of divergence errors in mhd simulations and consequences for numerical schemes, Commun. Appl. Math. Comput. Sci. 8 (2013), no. 1, 1-38.

[21] Contributions to the numerical simulation of gas, shallow water, and plasma flows - wave-wise treatment of order and viscosity, Habilitationsschrift, Brandenburgische Technische Universität, 2014.

[22] Friedemann Kemm, Yong-Joong Lee, Claus-Dieter Munz, and Rudolf Schneider, Divergence cleaning in finite-volume computations for electromagnetic wave propagations., Finite volumes for complex applications III. Problems and perspectives. Papers from the 3rd symposium of finite volumes for complex applications, Porquerolles, France, June 24-28 (Raphaéle Herbin and Dietmar Kröner, eds.), Hermes Penton Science, London, 2002, pp. 575-582 (English).

[23] Y.J. Lee, F. Kemm, C.-D. Munz, and R. Schneider, Physical symmetries and hyperbolic GLM divergence correction scheme for Maxwell and mhd equations, Hyperbolic Problems: Theory, Numerics, Applications (ThomasY. Hou and Eitan Tadmor, eds.), Springer Berlin Heidelberg, 2003, pp. 685-694 (English).

[24] Y.J. Lee, R. Schneider, C.-D. Munz, and F. Kemm, Hyperbolic GLM scheme for elliptic constraints in computational electromagnetics and $M H D$, Analysis and Numerics for Conservation Laws (Gerald Warnecke, ed.), Springer Berlin Heidelberg, 2005, pp. 385-404 (English).

[25] Barry Marder, A method incorporating gauß' law into electromagnetic PIC codes, J. Comput. Phys. 68 (1987), 48-55.

[26] Siddhartha Mishra and Eitan Tadmor, Constraint preserving schemes using potentialbased fluxes. I. multidimensional transport equations, Commun. Comput. Phys 9 (2010), 688-710.

[27] Siddhartha Mishra and Eitan Tadmor, Constraint preserving schemes using potentialbased fluxes. II: Genuinely multidimensional systems of conservation laws., SIAM J. Numer. Anal. 49 (2011), no. 3, 1023-1045 (English). 
[28] _ Constraint preserving schemes using potential-based fluxes. III: Genuinely multi-dimensional schemes for MHD equations., ESAIM, Math. Model. Numer. Anal. 46 (2012), no. 3, 661-680 (English).

[29] Claus-Dieter Munz, Pascal Omnes, Rudolf Schneider, Eric Sonnendrücker, and Ursula Voss, Divergence correction techniques for Maxwell solvers based on a hyperbolic model, J. Comput. Phys. 161 (1999), 484-511.

[30] Claus-Dieter Munz, Rodolf Schneider, and Ursula Voß, A finite volume method for the Maxwell equations in the time domain, SIAM J. Sci. Comput. 22 (2000), no. 2, 449-475.

[31] Claus-Dieter Munz, Rudolf Schneider, Eric Sonnendrücker, and Ursula Voss, Maxwell's equations when the charge conservation is not satisfied, C. R. Acad. Sci. Paris $\mathbf{3 2 8}$ (1999), no. S'erie I, 431-436.

[32] Kenneth G. Powell, Phil L. Roe, R. S̃. Myong, T. Gombosi, and D. de Zeeuw, An upwind scheme for magnetohydrodynamics, Workshop Methodes numeriques pour la M.H.D. (Luminy, France), 1995.

[33] Kenneth G. Powell, Philipp L. Roe, Timur Linde, Tamas I. Gombosi, and Darren L. DeZeeuw, A solution-adaptive upwind scheme for ideal magnetohydrodynamics, J. Comput. Phys. 154 (1999), 284-309.

[34] Shamsul Qamar and Gerald Warnecke, Application of space-time CE/SE method to shallow water magnetohydrodynamic equations., J. Comput. Appl. Math. 196 (2006), no. 1, 132-149 (English).

[35] Philip L. Roe, Approximate Riemann solvers, parameter vectors, and difference schemes, J. Comput. Phys. 43 (1981), no. 2, 357-372.

[36] James A. Rossmanith, A wave propagation method with constrained transport for ideal and shallow water magnetohydrodynamics, Ph.D. thesis, University of Washington, 2002.

[37] James A. Rossmanith, An unstaggered high-resolution constrained transport method for magnetohydrodynamic flows, SIAM J. Sci. Comput. 28 (2006), no. 5, 1766-1797.

[38] Hans De Sterck, Hyperbolic theory of the "shallow water" magnetohydrodynamics equations, Phys. Plasmas 8 (2001), no. 7, 3293-3304.

[39] _ Multi-dimensional upwind constrained transport on unstructured grids for "shallow water" magnetohydrodynamics, AIAA 2001-2623, 2001.

[40] Manuel Torrilhon, Locally divergence-preserving upwind finite volume schemes for magnetohydrodynamic equations, SIAM J. Sci. Comput. 26 (2005), no. 4, 1166-1191. 
[41] Manuel Torrilhon and Michael Fey, Constraint-preserving upwind methods for multidimensional advection equations, SIAM J. Numer. Anal. 42 (2004), no. 4, 1694-1728.

[42] I. Toumi, A weak formulation of Roe's approximate Riemann solver, Journal of Computational Physics 102 (1992), no. 2, 360 - 373.

[43] Knut Waagan, A positive MUSCL-Hancock scheme for ideal magnetohydrodynamics, J. Comput. Phys. 228 (2009), no. 23, 8609-8626. 